\title{
The Marshall-Olkin-Gompertz-G family of distributions: properties and applications
}

\author{
Fastel Chipepa $a^{\mathrm{a}, \mathrm{b}, *}$, Broderick Oluyede ${ }^{\mathrm{a}}$ \\ ${ }^{a}$ Department of Mathematical Statistics, Botswana International University of Science and Technology, P. Bag 16, Palapye, Botswana. \\ ${ }^{b}$ Department of Applied Mathematics and Statistics, Midlands State University, P. Bag 9055, Gweru, Zimbabwe.
}

\begin{abstract}
We develop a new generalized family of the Gompertz-G distribution, namely, the Marshall-Olkin-Gompertz-G distribution. Statistical properties of the new proposed model are presented. Some special cases of the new family of distributions are presented. Maximum likelihood estimates of the model parameters are also determined. A simulation study was conducted to assess the performance of the maximum likelihood estimates. Applications to demonstrate the usefulness of the Marshall-OlkinGompertz-Weibull distribution to real data examples are provided.
\end{abstract}

Keywords: Gompertz-G distribution, Marshall-Olkin-G distribution, maximum likelihood estimation.

2020 MSC: 62E99, 60E05.

(C)2021 All rights reserved.

\section{Introduction}

There is remarkable increase in the acceptability of generalized distributions over the past two decades. The motivation for the development of generalized distributions is their applicability to model heavy tailed data which is a prevalent phenomena in many lifetime analysis. Other areas of application of generalized distributions include finance, economics, hydrology and physics. Several attempts for generalizing classical distributions have been made. Earlier attempts include work by Gurvich et al. [22], Marshall and Olkin [26], Gupta and Kundu [21] and Eugene et al. [17].

Consequently, many generators for generalizing classical distributions have been proposed, these include gamma-G type I, type 2 and type 3 by Zografos and Balakrishnan [36], Ristić and Balakrishnan [30], Torabi and Hedesh [34], respectively, Weibull-G by Bourguignon et al. [6], Kumaraswamy-G (Kw-G) by Cordeiro et al. [14], type I half logistic-G family by Cordeiro et al. [12], odd log-logistic-G by Gleaton and Lynch [18], Gompertz-G by Alizadeh et al. [2], to mention a few.

Of interest in this paper are the generalized distributions by Alizadeh et al. [2], and Marshall and Olkin [26]. The Gompertz-G (Gom-G) distribution have cumulative distribution function (cdf) and probability density function (pdf) given by

$$
\mathrm{F}_{\mathrm{Gom}-\mathrm{G}}(x ; \theta, \gamma, \xi)=1-e^{\frac{\theta}{\gamma}\left(1-[1-\mathrm{G}(x ; \xi)]^{-\gamma}\right)}
$$

\footnotetext{
*Corresponding author

Email addresses: chipepaf@staff.msu.ac.zw (Fastel Chipepa), oluyedeo@biust.ac.bw (Broderick Oluyede)

doi: $10.22436 /$ jnsa.014.04.05
}

Received: 2020-10-08 Revised: 2020-10-29 Accepted: 2020-12-02 
and

$$
f_{\mathrm{Gom}-\mathrm{G}}(x ; \theta, \gamma, \xi)=\theta g(x ; \xi)[1-\mathrm{G}(x ; \xi)]^{-\gamma-1} e^{\frac{\theta}{\gamma}\left(1-[1-G(x ; \xi)]^{-\gamma}\right)},
$$

respectively, for $\theta, \gamma>0$, and parameter vector $\xi$. The Marshall-Olkin-G distribution by Marshall and Olkin [26] have cdf given by

$$
F_{M O-G}(x ; \xi)=\frac{G(x ; \xi)}{1-\bar{\delta} \bar{G}(x ; \xi)}
$$

where $\delta$ is the tilt parameter, $\overline{\mathrm{G}}(x ; \xi)=1-\mathrm{G}(x ; \xi)$ is the survival function, and parameter vector $\xi$ from the baseline distribution.

We generalize the Gom-G distribution via the Marshall-Olkin generator to produce a new family of distributions called the Marshall-Olkin-Gompertz-G (MO-Gom-G) family of distributions. The MO-Gom$\mathrm{G}$ distribution gives improved flexibility in data fitting (i.e., the model applies to heavy tailed, right or left-skewed data sets) and improved shapes of the hazard rate function (monotonic, bathtub, upside bathtub and upside bathtub followed by bathtub shapes) compared to the Gompertz-G distribution. The proposed distribution has a desirable tractability property and can be expressed as an infinite linear combination of the exponentiated-G distribution. This property makes the derivation of the other statistical properties possible. Other generalizations of the Gompertz distribution available in the literature includes the exponentiated Gompertz generated family by Cordeiro et al. [13], beta-Gompertz distribution by Jafari et al. [23], odd log-logistic Gompertz and odd log-logistic generalized Gompertz distributions by Alizadeh et al. ([1,3]) and new extended generalized Gompertz distribution by Karamikabir et al. [24].

The paper is organized as follows. Section 2, presents the proposed model and some statistical properties. Estimation is presented in Section 3. Some special cases of the new family of distributions are presented in Section 4. A simulation study is presented in Section 5. Section 6 provides some applications to show the usefulness of the new proposed model followed by concluding remarks in Section 7.

\section{The model and statistical properties}

In this section, we develop the MO-Gom-G family of distributions. We use the generalizations by Marshall and Olkin [26] and Alizadeh et al. [2] given respectively by equations (1.3) and (1.1). The cdf, pdf, and hrf of the MO-Gom-G family of distributions are given by

$$
\begin{aligned}
& F_{M O-G o m-G}(x ; \theta, \gamma, \delta, \xi)=\frac{1-e^{\frac{\theta}{\gamma}\left(1-[1-G(x ; \xi)]^{-\gamma}\right)}}{1-\bar{\delta}\left(e^{\frac{\theta}{\gamma}\left(1-[1-G(x ; \xi)]^{-\gamma}\right)}\right)}, \\
& f_{M O-G o m-G}(x ; \theta, \gamma, \delta, \xi)=\frac{\delta \theta g(x ; \xi)[1-G(x ; \xi)]^{-\gamma-1} e^{\frac{\theta}{\gamma}\left(1-[1-G(x ; \xi)]^{-\gamma}\right)}}{\left(1-\bar{\delta}\left(e^{\frac{\theta}{\gamma}\left(1-[1-G(x ; \xi)]^{-\gamma}\right)}\right)\right)^{2}}, \\
& h(x ; \theta, \gamma, \delta, \xi)=\frac{\delta \theta g(x ; \xi)[1-G(x ; \xi)]^{-\gamma-1} e^{\frac{\theta}{\gamma}\left(1-[1-G(x ; \xi)]^{-\gamma}\right)}}{\left(1-\bar{\delta}\left(e^{\frac{\theta}{\gamma}\left(1-[1-G(x ; \xi)]^{-\gamma}\right)}\right)\right)^{2}} \\
& \times\left[1-\frac{1-e^{\frac{\theta}{\gamma}\left(1-[1-G(x ; \xi)]^{-\gamma}\right)}}{1-\bar{\delta}\left(e^{\frac{\theta}{\gamma}\left(1-[1-G(x ; \xi)]^{-\gamma}\right)}\right)}\right]^{-1},
\end{aligned}
$$

respectively, for $\theta, \gamma, \delta>0, \bar{\delta}=1-\delta$ and a parameter vector $\xi$. If a random variable $\mathrm{X}$ has the MO-Gom-G distribution, we write $\mathrm{X} \sim \mathrm{MO}-\mathrm{Gom}-\mathrm{G}(\theta, \gamma, \delta, \xi)$.

\subsection{Quantile function}

We invert the cdf of the MO-Gom-G family of distributions to obtain the quantile function as follows:

$$
\frac{1-e^{\frac{\theta}{\gamma}\left(1-[1-G(x ; \xi)]^{-\gamma}\right)}}{1-\bar{\delta}\left(e^{\frac{\theta}{\gamma}\left(1-[1-G(x ; \xi)]^{-\gamma}\right)}\right)}=u,
$$


for $0<u<1$. Note that

$$
\log \left[\frac{1-u}{1-u \bar{\delta}}\right]=\frac{\theta}{\gamma}\left(1-[1-G(x ; \xi)]^{-\gamma}\right)
$$

so that

$$
\mathrm{G}(x ; \xi)=1-\left(1-\frac{\gamma}{\theta} \log \left[\frac{1-u}{1-u \bar{\delta}}\right]\right)^{-1 / \gamma} .
$$

Therefore, the solution to the non-linear equation

$$
\mathrm{Q}_{x}(\mathrm{u})=\mathrm{G}^{-1}\left[1-\left(1-\frac{\gamma}{\theta} \log \left[\frac{1-\mathrm{u}}{1-\mathrm{u} \bar{\delta}}\right]\right)^{-1 / \gamma}\right]
$$

gives the quantile values of the MO-Gom-G family of distributions.

\subsection{Stochastic orders}

In this subsection, we present stochastic orders for the MO-Gom-G family of distributions. Suppose we have two random variables $X$ and $Z$ with distribution functions $F_{X}(r)$ and $F_{Z}(r)$, respectively, and $\bar{F}_{X}(r)=1-F_{X}(r)$ the survival function, $X$ is stochastically smaller than $Z$ if $\bar{F}_{X}(r) \leqslant \bar{F}_{Z}(r)$ for all $r$ or $F_{X}(r) \geqslant F_{Z}(r)$ for all $r$. This is denoted by $X<_{s} Z$. Hazard rate order and likelihood ratio order are stronger and are given by $X<_{h r} Z$ if $h_{X}(r) \geqslant h_{Z}(r)$ for all $r$, and $X<_{l r} Z$ if $\frac{f_{X}(r)}{f_{Z}(r)}$ is decreasing in $r$. From Shaked and Shanthikumar [31], we know that $X<_{l r} Z \Rightarrow X<_{h r} Z \Rightarrow X<_{s} Z$.

Theorem 2.1. Let $\mathrm{X}_{1} \sim \mathrm{MO}-\mathrm{Gom}-\mathrm{G}\left(\theta, \gamma, \delta_{1}, \xi\right)$ and $\mathrm{X}_{2} \sim \mathrm{MO}-\mathrm{Gom}-\mathrm{G}\left(\theta, \gamma, \delta_{2}, \xi\right)$. If $\delta_{1} \leqslant \delta_{2}$, then $\frac{\mathrm{f}\left(\mathrm{x} ; \theta, \gamma, \delta_{1}, \xi\right)}{\mathrm{f}\left(\mathrm{x} ; \theta, \gamma, \delta_{2}, \xi\right)}$ is decreasing.

Proof. Note that

$$
\frac{f\left(x ; \theta, \gamma, \delta_{1}, \xi\right)}{f\left(x ; \theta, \gamma, \delta_{2}, \xi\right)}=\frac{\delta_{1}}{\delta_{2}}\left(\frac{1-\overline{\delta_{1}}\left(e^{\frac{\theta}{\gamma}\left(1-[1-G(x ; \xi)]^{-\gamma}\right)}\right)}{1-\overline{\delta_{2}}\left(e^{\frac{\theta}{\gamma}\left(1-[1-G(x ; \xi)]^{-\gamma}\right)}\right)}\right)^{2}
$$

so that

$$
\begin{aligned}
\frac{\partial}{\partial x}\left(\frac{f\left(x ; \theta, \gamma, \delta_{1}, \xi\right)}{f\left(x ; \theta, \gamma, \delta_{2}, \xi\right)}\right)= & \frac{\delta_{1}}{\delta_{2}}\left(\delta_{1}-\delta_{2}\right) \frac{1-\overline{\delta_{1}}\left(e^{\frac{\theta}{\gamma}\left(1-[1-G(x ; \xi)]^{-\gamma}\right)}\right)}{\left[1-\overline{\delta_{2}}\left(e^{\frac{\theta}{\gamma}\left(1-[1-G(x ; \xi)]^{-\gamma}\right)}\right)\right]^{3}} \\
& \left.\times \theta g(x ; \xi)[1-G(x ; \xi)]^{-\gamma-1} e^{\frac{\theta}{\gamma}\left(1-[1-G(x ; \xi)]^{-\gamma}\right.}\right)
\end{aligned}
$$

which is $\leqslant 0$ if $\delta_{1} \leqslant \delta_{2}$. Therefore, $X_{1}<_{l r} X_{2}, X_{1}<_{h r} X_{2}$ and $X_{1}<_{s} X_{2}$. Hence, the random variables $X_{1}$ and $X_{2}$ are stochastically ordered.

\subsection{Linear representation}

We derive the linear representation of the MO-Gom-G family of distributions in this subsection. We make use of the general results by Barreto-Souza et al. [5]. Considering

$$
f_{M O-G o m-G}(x ; \theta, \gamma, \delta, \xi)=\frac{\delta f_{\text {Gom-G }}(x ; \theta, \gamma, \xi)}{\left(1-\bar{\delta} \bar{F}_{\text {Gom-G }}(x ; \theta, \gamma, \xi)\right)^{2}},
$$

we can write equation (2.1) as

$$
f_{\text {MO-Gom-G }}(x ; \theta, \gamma, \delta, \xi)=\frac{f_{\text {Gom-G }}(x ; \theta, \gamma, \xi)}{\delta\left[1-\frac{\delta-1}{\delta} F_{G o m-G}(x ; \theta, \gamma, \xi)\right]^{2}},
$$


where $f_{G o m-G}(x ; \theta, \gamma, \xi)$ and $F_{G o m-G}(x ; \theta, \gamma, \xi)$ are as given in equations (1.2) and (1.1), respectively. We also apply the series expansion

$$
(1-z)^{-k}=\sum_{j=0}^{\infty} \frac{\Gamma(k+j)}{\Gamma(k) j !} z^{j},
$$

which is valid for $|z|<1$ and $k>0$. If $\delta \in(0,1)$, we obtain

$$
f_{\text {MO-Gom-G }}(x ; \theta, \delta, \gamma, \xi)=f_{\text {Gom-G }}(x ; \theta, \gamma, \xi) \sum_{j=0}^{\infty} \sum_{k=0}^{j} w_{j, k} F_{G o m-G}(x ; \theta, \gamma, \xi)^{j-k},
$$

where $w_{j, k}=w_{j, k}(\delta)=\delta(j+1)(1-\delta)^{j}(-1)^{j-k}\left(\begin{array}{l}j \\ k\end{array}\right)$. For $\delta>1$, we have

$$
\mathrm{f}_{\text {MO-Gom-G }}(x ; \theta, \delta, \gamma, \xi)=f_{\text {Gom-G }}(x ; \theta, \gamma, \xi) \sum_{j=0}^{\infty} \nu_{j} F_{G o m-G}^{j}(x ; \theta, \gamma, \xi),
$$

where $v_{j}=v_{j}(\delta)=\frac{(j+1)(1-1 / \delta)}{\delta}$. Thus, for $\delta \in(0,1)$, equation (2.1) becomes

$$
\begin{aligned}
f_{\text {MO-Gom-G }}(x ; \theta, \delta, \gamma, \xi)= & \theta g(x ; \xi)[1-G(x ; \xi)]^{-\gamma-1} e^{\frac{\theta}{\gamma}\left(1-[1-G(x ; \xi)]^{-\gamma}\right)} \\
& \times \sum_{j=0}^{\infty} \sum_{k=0}^{j} w_{j, k}\left[1-e^{\frac{\theta}{\gamma}\left(1-[1-G(x ; \xi)]^{-\gamma}\right)}\right]^{j-k}
\end{aligned}
$$

Using the following series expansions

$$
\begin{aligned}
{\left[1-e^{\left.\frac{\theta}{\gamma}\left(1-[1-G(x ; \xi)]^{-\gamma}\right)\right]^{j-k}}\right.} & =\sum_{m=0}^{\infty}(-1)^{m}\left(\begin{array}{c}
j-k \\
m
\end{array}\right) e^{\frac{\theta}{\gamma} m\left(1-[1-G(x ; \xi)]^{-\gamma}\right)}, \\
e^{\frac{\theta}{\gamma}(m+1)\left(1-[1-G(x ; \xi)]^{-\gamma}\right)} & =\sum_{n=0}^{\infty} \frac{\left(\frac{\theta}{\gamma}\right)^{n}(m+1)^{n}\left(1-[1-G(x ; \xi)]^{-\gamma}\right)^{n}}{n !} \\
\left(1-[1-G(x ; \xi)]^{-\gamma}\right)^{n} & =\sum_{p=0}^{\infty}(-1)^{p}\left(\begin{array}{c}
n \\
p
\end{array}\right)[1-G(x ; \xi)]^{-\gamma p}, \\
{[1-G(x ; \xi)]^{-\gamma(p+1)-1} } & =\sum_{q=0}^{\infty}\left(\begin{array}{c}
-\gamma(p+1)-1 \\
q
\end{array}\right) G^{q}(x ; \xi),
\end{aligned}
$$

we have for $\delta \in(0,1)$,

$$
\begin{aligned}
f_{\text {MO-Gom-G }}(x ; \theta, \gamma, \delta, \xi)= & \sum_{j, m, n, p, q=0}^{\infty} \sum_{k=0}^{j} \frac{(-1)^{p+m} \theta^{n+1}(m+1)^{n}}{\gamma^{n} n !} w_{j, k}\left(\begin{array}{c}
j-k \\
m
\end{array}\right)\left(\begin{array}{c}
n \\
p
\end{array}\right) \\
& \times\left(\begin{array}{c}
-\gamma(p+1)-1 \\
q
\end{array}\right) g(x ; \xi) G^{q}(x ; \xi)=\sum_{q=0}^{\infty} w_{q}^{*} g_{q}(x ; \xi) .
\end{aligned}
$$

It follows that for $\delta \in(0,1)$, the MO-Gom-G family of distributions can be expressed as a linear combination of the exponentiated-G (Exp-G) distribution with power parameter $q$ and linear component

$$
w_{q}^{*}=\sum_{j, m, n, p}^{\infty} \sum_{k=0}^{j} \frac{(-1)^{p+m} \theta^{n+1}(m+1)^{n}}{(q+1) \gamma^{n} n !} w_{j, k}\left(\begin{array}{c}
j-k \\
m
\end{array}\right)\left(\begin{array}{c}
n \\
p
\end{array}\right)\left(\begin{array}{c}
-\gamma(p+1)-1 \\
q
\end{array}\right)
$$


and $g_{q}(x ; \xi)=(q+1) g(x ; \xi) G^{q}(x ; \xi)$.

Furthermore, for $\delta>1$, equation (2.1) can be written as

$$
\mathrm{f}_{\text {MO-Gom-G }}(x ; \theta, \gamma, \delta, \xi)=\theta g(x ; \xi)\left[1-G(x ; \xi)^{-\gamma-1}\right] e^{\frac{\theta}{\gamma}\left(1-[1-G(x ; \xi)]^{-\gamma}\right)} \sum_{j=0}^{\infty} v_{j}\left[1-e^{\frac{\theta}{\gamma}\left(1-[1-G(x ; \xi)]^{-\gamma}\right)}\right]^{j} .
$$

Using the series expansion

$$
\left[1-e^{\left.\frac{\theta}{\gamma}\left(1-[1-G(x ; \xi)]^{-\gamma}\right)\right]^{j}}=\sum_{m=0}^{\infty}(-1)^{m} e^{\frac{\theta}{\gamma}\left(1-[1-G(x ; \xi)]^{-\gamma}\right) m}\left(\begin{array}{c}
j \\
m
\end{array}\right),\right.
$$

equations (2.3), (2.4), and (2.5), yields

$$
\begin{aligned}
f_{\text {MO-Gom-G }}(x ; \theta, \gamma, \delta, \xi)= & \sum_{j, m, n, p, q=0}^{\infty} \frac{(-1)^{m+p} \theta^{n+1}(m+1)^{n}}{\gamma^{n} n !} v_{j}\left(\begin{array}{c}
j \\
m
\end{array}\right)\left(\begin{array}{l}
n \\
p
\end{array}\right) \\
& \times\left(\begin{array}{c}
-\delta(p+1)-1 \\
q
\end{array}\right) g(x ; \xi) G^{q}(x ; \xi)=\sum_{q=0}^{\infty} v_{q}^{*} g_{q}(x ; \xi) .
\end{aligned}
$$

Also, for $\delta>1$, the MO-Gom-G family of distributions can be expressed as a linear combination of the Exp-G distribution with power parameter $q$ and linear component

$$
v_{q}^{*}=\sum_{j, m, n, p=0}^{\infty} \frac{(-1)^{m+p} \theta^{n+1}(m+1)^{n}}{(q+1) \gamma^{n} n !} v_{j}\left(\begin{array}{c}
j \\
m
\end{array}\right)\left(\begin{array}{c}
n \\
p
\end{array}\right)\left(\begin{array}{c}
-\delta(p+1)-1 \\
q
\end{array}\right) .
$$

\subsection{Moments and generating function}

Let $X \sim M O-G o m-G(\theta, \gamma, \delta, \xi)$, then the $r^{\text {th }}$ moment can be obtained from equations (2.6) and (2.8), since the MO-Gom-G family of distributions can be expressed as a linear combination of Exp-G distribution.

- For $\delta \in(0,1)$,

$$
E\left(X^{r}\right)=\sum_{q=0}^{\infty} w_{q}^{*} E\left(H_{q}^{r}\right)
$$

where $w_{q}^{*}$ is as defined in equation (2.7) and $E\left(H_{q}^{r}\right)$ denotes the $r^{\text {th }}$ moment of $H_{q}$ which follows an Exp-G distribution with power parameter $\mathrm{q}$.

- For $\delta>1$

$$
\mathrm{E}\left(\mathrm{X}^{\mathrm{r}}\right)=\sum_{\mathrm{q}=0}^{\infty} v_{\mathrm{q}}^{*} \mathrm{E}\left(\mathrm{H}_{\mathrm{q}}^{\mathrm{r}}\right)
$$

where $v_{q}^{*}$ is as defined in equation (2.9) and $E\left(H_{q}^{r}\right)$ denotes the $r^{\text {th }}$ moment of $H_{q}$ which follows an Exp-G distribution with power parameter $\mathrm{q}$. The incomplete moments can be obtained as follows:

- For $\delta \in(0,1)$

$$
\mathrm{I}_{X}(\mathrm{t})=\int_{0}^{\mathrm{t}} x^{\mathrm{s}} \mathrm{f}_{\mathrm{MO}-\mathrm{Gom}-\mathrm{G}}(x ; \theta, \gamma, \delta, \xi) \mathrm{d} x=\sum_{\mathrm{q}=0}^{\infty} w_{\mathrm{q}}^{*} \mathrm{I}_{\mathrm{q}}(\mathrm{t}),
$$

where $I_{q}(t)=\int_{0}^{t} x^{r} g_{q}(x ; \xi) d x$ and $w_{q}^{*}$ is as defined in equation (2.7).

- For $\delta>1$

$$
I_{X}(t)=\int_{0}^{t} x^{s} f_{M O-G o m-G}(x ; \theta, \gamma, \delta, \xi) d x=\sum_{q=0}^{\infty} v_{q}^{*} I_{q}(t),
$$


where $I_{q}(t)=\int_{0}^{t} x^{r} g_{q}(x ; \xi) d x$ and $v_{q}^{*}$ is as defined in equation (2.9). The moment generating function (mgf) of $X$ is given by:

- For $\delta \in(0,1)$

$$
M_{X}(t)=\sum_{q=0}^{\infty} w_{q}^{*} E\left(e^{t H_{q}}\right),
$$

where $E\left(e^{t H_{q}}\right)$ is the mgf of the Exp-G distribution with power parameter $q$ and $w_{q}^{*}$ is as defined in equation (2.7).

- For $\delta>1$

$$
M_{X}(t)=\sum_{q=0}^{\infty} v_{q}^{*} E\left(e^{t H_{q}}\right),
$$

where $E\left(e^{t H_{q}}\right)$ is the mgf of the Exp-G distribution with power parameter $q$ and $v_{q}^{*}$ is as defined in equation (2.9).

\subsection{Entropy}

Entropy is a measure of variation of uncertainty for a random variable $X$ with pdf $g(x)$. The two common measures of entropy are Shannon entropy [32] and Rényi entropy [29]. Shannon entropy is a special case of Rényi entropy. So in this paper we derive Rényi entropy of the MO-Gom-G family of distributions. By definition, Rényi entropy is given by

$$
I_{R}(v)=(1-v)^{-1} \log \left[\int_{0}^{\infty} g^{v}(x) d x\right]
$$

where $v>0$ and $v \neq 1$. Using generalized binomial expansion (2.2), we get, for $\delta \in(0,1)$

$$
f_{\text {MO-Gom-G }}^{v}(x ; \theta, \delta, \gamma, \xi)=\frac{\delta^{v} f_{\text {Gom-G }}^{v}(x ; \theta, \gamma, \xi)}{\Gamma(2 v)} \sum_{j=0}^{\infty}(1-\delta)^{j} \Gamma(2 v+j) \frac{\left[1-F_{\text {Gom-G }}(x ; \theta, \gamma, \xi)\right]^{j}}{j !}
$$

and for $\delta>1$

$$
f_{\text {MO-Gom-G }}^{v}(x ; \theta, \delta, \gamma, \xi)=\frac{f_{\text {Gom-G }}^{v}(x ; \theta, \gamma, \xi)}{\delta^{v} \Gamma(2 v)} \sum_{j=0}^{\infty}(\delta-1)^{j} \Gamma(2 v+j) \frac{F_{G o m-G}^{j}(x ; \theta, \gamma, \xi)}{j !} .
$$

Thus, Rényi entropy for $\delta \in(0,1)$ and $\delta>1$ are given by

$$
I_{R}(v)=(1-v)^{-1} \log \left(\sum_{j=0}^{\infty} e_{j} \int_{0}^{\infty} f_{G o m-G}^{v}(x ; \theta, \gamma, \xi)\left(1-F_{G o m-G}(x ; \theta, \gamma, \xi)\right)^{j} d x\right)
$$

and

$$
I_{R}(v)=(1-v)^{-1} \log \left(\sum_{j=0}^{\infty} h_{j} \int_{0}^{\infty} f_{G o m-G}^{v}(x ; \theta, \gamma, \xi) F_{G o m-G}^{j}(x ; \theta, \gamma, \xi) d x\right),
$$

where

$$
e_{j}=e_{j}(\delta)=\frac{\delta^{v}(1-\delta)^{j} \Gamma(2 v+j)}{\Gamma(2 v) j !} \quad \text { and } \quad h_{j}=h_{j}(\delta)=\frac{(\delta-1)^{j} \Gamma(2 v+j)}{\delta^{v+j} \Gamma(2 v) j !} .
$$

Now, for $\delta \in(0,1)$,

$$
f_{\text {Gom-G }}^{\nu}(x ; \theta, \gamma, \xi)\left(1-F_{\text {Gom }-G}(x ; \theta, \gamma, \xi)\right)^{j}=\theta^{v} g^{\nu}(x, \xi)[1-G(x ; \xi)]^{(-\gamma-1) v} e^{\frac{\theta}{\gamma}(j+v)\left(1-[1-G(x ; \xi)]^{-\gamma}\right)} .
$$


Using the series expansion

$$
e^{\frac{\theta}{\gamma}(j+v)\left(1-[1-G(x ; \xi)]^{-\gamma}\right)}=\sum_{n=0}^{\infty} \frac{\left(\frac{\theta}{\gamma}\right)^{n}(j+v)^{n}\left(1-[1-G(x ; \xi)]^{-\gamma}\right)^{n}}{n !},
$$

as well as equations (2.4) and (2.5), we get

$$
f_{\text {Gom-G }}^{v}(x ; \theta, \gamma, \xi)\left(1-F_{G o m-G}(x ; \theta, \gamma, \xi)\right)^{j}=\sum_{n, p, q=0}^{\infty} \frac{(-1)^{p} \theta^{\theta+v}(j+v)^{n}}{\gamma^{n} n !}\left(\begin{array}{l}
n \\
p
\end{array}\right)\left(\begin{array}{c}
-\delta(p+v)-v \\
q
\end{array}\right) g(x ; \xi) G^{q}(x ; \xi) .
$$

Therefore, for $\delta \in(0,1)$,

$$
\begin{aligned}
I_{R}(v) & =(1-v)^{-1} \log \left[\sum_{j, n, p, q}^{\infty} \frac{(-1)^{p} \theta^{\theta+v}(j+v)^{n}}{\gamma^{n} n ! j !}\left(\begin{array}{c}
n \\
p
\end{array}\right)\left(\begin{array}{c}
-\delta(p+v)-v \\
q
\end{array}\right) \int_{0}^{\infty} g(x ; \xi) G^{q}(x ; \xi) d x\right] \\
& =(1-v)^{-1} \log \left[\sum_{q=0}^{\infty} \eta_{q}^{*} e^{(1-v) I_{R E G}}\right],
\end{aligned}
$$

for $v>0, v \neq 1$, where $I_{R E G}=\frac{1}{1-v} \log \left[\int_{0}^{\infty}\left(\left[\frac{q}{v}+1\right](G(x ; \xi))^{\frac{q}{v}} g(x ; \xi)\right)^{v} d x\right]$ is Rényi entropy of Exp-G distribution with power parameter $\left(\frac{q}{v}\right)$, and

$$
\eta_{\mathrm{q}}^{*}=\sum_{j, n, p=0}^{\infty} \frac{(-1)^{p} \theta^{\theta+v}(j+v)^{n}}{\gamma^{n} n ! j !}\left(\begin{array}{l}
n \\
p
\end{array}\right)\left(\begin{array}{c}
-\delta(p+v)-v \\
q
\end{array}\right) \frac{1}{\left[1+\frac{q}{v}\right]^{v}}
$$

Also, for $\delta>1$,

$$
\begin{aligned}
f_{G o m-G}^{\nu}(x ; \theta, \gamma, \xi) F_{G o m-G}^{j}(x ; \theta, \gamma, \xi)= & \theta^{\nu} g^{\nu}(x, \xi)[1-G(x ; \xi)]^{(-\gamma-1) \nu} \\
& \times e^{\frac{\theta}{\gamma}\left(1-[1-G(x ; \xi)]^{-\gamma}\right)^{v}}\left[1-e^{\frac{\theta}{\gamma}\left(1-[1-G(x ; \xi)]^{-\gamma}\right)}\right]^{j} .
\end{aligned}
$$

Using equations (2.3), the series expansion

$$
e^{\frac{\theta}{\gamma}(m+v)\left(1-[1-G(x ; \xi)]^{-\gamma}\right)^{v}}=\sum_{n=0}^{\infty}\left(\frac{\theta}{\gamma}\right)^{n}\left(1-[1-G(x ; \xi)]^{-\gamma}\right)^{n},
$$

as well as equations (2.4) and (2.5), we get

$$
\begin{aligned}
f_{G o m-G}^{v}(x ; \theta, \gamma, \xi) F_{G o m-G}^{j}(x ; \theta, \gamma, \xi)= & \sum_{m, n, p, q=0}^{\infty} \frac{(-1)^{m+p} \theta^{n+v}(m+v)^{n}}{\gamma^{n} n !}\left(\begin{array}{c}
j \\
m
\end{array}\right)\left(\begin{array}{c}
n \\
p
\end{array}\right) \\
& \times\left(\begin{array}{c}
-\gamma(p+v)-v \\
q
\end{array}\right) g^{v}(x ; \xi) G^{q}(x ; \xi) .
\end{aligned}
$$

Hence, for $\delta>1$,

$$
\begin{aligned}
I_{R}(v)= & (1-v)^{-1} \log \left[\sum_{j, m, n, p, q=0}^{\infty} \frac{(-1)^{m+p} \theta^{n+v}(m+v)^{n}}{\gamma^{n} n ! j !}\left(\begin{array}{c}
j \\
m
\end{array}\right)\left(\begin{array}{l}
n \\
p
\end{array}\right)\right. \\
& \left.\times\left(\begin{array}{c}
-\gamma(p+v)-v \\
q
\end{array}\right) \int_{0}^{\infty} g^{v}(x ; \xi) G^{q}(x ; \xi) d x\right]=(1-v)^{-1} \log \left[\sum_{q=0}^{\infty} \eta_{q} e^{(1-v) I_{R E G}}\right],
\end{aligned}
$$


for $v>0, v \neq 1$, where $I_{R E G}=\frac{1}{1-v} \log \left[\int_{0}^{\infty}\left(\left[\frac{q}{v}+1\right](G(x ; \xi))^{\frac{q}{v}} g(x ; \xi)\right)^{v} d x\right]$ is Rényi entropy of Exp-G distribution with power parameter $\left(\frac{\mathrm{q}}{v}\right)$, and

$$
\eta_{q}=\sum_{j, m, n, p=0}^{\infty} \frac{(-1)^{m+p} \theta^{n+v}(m+v)^{n}}{\gamma^{n} n ! j !}\left(\begin{array}{c}
j \\
m
\end{array}\right)\left(\begin{array}{l}
n \\
p
\end{array}\right)\left(\begin{array}{c}
-\gamma(p+v)-v \\
q
\end{array}\right) \frac{1}{\left[1+\frac{q}{v}\right]^{v}} .
$$

It follows that Rényi entropy of the MO-Gom-G family of distributions can be derived directly from Rényi entropy of the Exp-G distribution.

\subsection{Distribution of order statistics}

Suppose that $X_{1}, X_{2}, \ldots, X_{n}$ are independent and identically distributed (i.i.d) random variables distributed according to equation (2.1), then the pdf of the $i^{\text {th }}$ order statistic is given by

$$
f_{i: n}(x)=\delta n ! f_{G o m-G}(x ; \theta, \gamma, \xi) \sum_{l=0}^{n-i} \frac{(-1)^{l}}{(i-1) !(n-i) !} \frac{F_{G o m-G}^{l+i-1}(x ; \theta, \gamma, \xi)}{\left[1-\bar{\delta} F_{G o m-G}(x ; \theta, \gamma, \xi)\right]^{l+i-1}} .
$$

If $\delta \in(0,1)$, we have

$$
f_{i: n}(x)=f_{G o m-G}(x ; \theta, \gamma, \xi) \sum_{j=0}^{\infty} \sum_{l=0}^{n-i} \sum_{k=0}^{j} u_{j, l, k} F_{G o m-G}^{j+l-k+i-1}(x ; \theta, \gamma, \xi),
$$

where

$$
u_{j, l, k}=u_{j, l, k}(\delta)=\frac{\delta n !(-1)^{l}(1-\delta)^{j}(-1)^{j-k}}{(i-1) !(n-i) !}\left(\begin{array}{l}
j \\
k
\end{array}\right)\left(\begin{array}{c}
l+i+j \\
j
\end{array}\right) .
$$

For $\delta>1$,

$$
f_{i: n}(x)=f_{G o m-G}(x ; \theta, \gamma, \xi) \sum_{j=0}^{\infty} \sum_{l=0}^{n-i} c_{j, l} F_{G o m-G}^{j+l+i-1}(x ; \theta, \gamma, \xi),
$$

where

$$
c_{j, l}=c_{j, l}(\delta)=\frac{(-1)^{l}(\delta-1)^{j} n !}{\delta^{l+j+i}(i-1) !(n-i) !}\left(\begin{array}{c}
l+\mathfrak{i}+j \\
j
\end{array}\right) .
$$

Therefore, for $\delta \in(0,1)$, we get

$$
\begin{aligned}
f_{i: n}= & \theta g(x ; \xi)[1-G(x ; \xi)]^{-\gamma-1} e^{\frac{\theta}{\gamma}\left(1-\left[1-G\left(x_{i} ; \xi\right)\right]^{-\gamma}\right)} \\
& \times \sum_{j=0}^{\infty} \sum_{l=0}^{n-i} \sum_{k=0}^{j} u_{j, l, k}\left(1-e^{\frac{\theta}{\gamma}\left(1-\left[1-G\left(x_{i} ; \xi\right)\right]^{-\gamma}\right)}\right)^{j+l-k+i-1} .
\end{aligned}
$$

Using the generalized binomial series expansion

$$
\left(1-\left[1-\mathrm{G}\left(x_{i} ; \xi\right)\right]^{-\gamma}\right)^{j+l-k+i-1}=\sum_{m=0}^{\infty}(-1)^{m}\left(\begin{array}{c}
j+l-k+i-1 \\
m
\end{array}\right) e^{\frac{\theta m}{\gamma}\left(1-\left[1-G\left(x_{i} ; \xi\right)\right]^{-\gamma}\right)},
$$

as well as equations (2.3), (2.4), and (2.5), we can write

$$
\begin{aligned}
f_{i: n}(x)= & \sum_{j, m, n, p, q=0}^{\infty} \sum_{l=0}^{n-i} \sum_{k=0}^{j} \frac{(-1)^{m+p} \theta^{n+1}(m+1)^{n}}{\gamma^{n} n !} u_{j, l, k}\left(\begin{array}{c}
j+l-k+i-1 \\
m
\end{array}\right)\left(\begin{array}{l}
n \\
p
\end{array}\right) \\
& \times\left(\begin{array}{c}
-\gamma(p+1)-1 \\
q
\end{array}\right) g(x ; \xi)[G(x ; \xi)]^{q}=\sum_{q=0}^{\infty} u_{q}^{*} g_{q}(x ; \xi),
\end{aligned}
$$


where $g_{q}(x ; \xi)=(q+1) g(x ; \xi)[G(x ; \xi)]^{q}$ is an Exp-G distribution with power parameter $q$ and

$$
u_{q}^{*}=\sum_{j, m, n, p}^{\infty} \sum_{l=0}^{n-i} \sum_{k=0}^{j} \frac{(-1)^{m+p} \theta^{n+1}(m+1)^{n}}{(q+1) \gamma^{n} n !} u_{j, l, k}\left(\begin{array}{c}
j+l-k+i-1 \\
m
\end{array}\right)\left(\begin{array}{l}
n \\
p
\end{array}\right)\left(\begin{array}{c}
-\gamma(p+1)-1 \\
q
\end{array}\right) .
$$

Furthermore, for $\delta>1$, we write

$$
f_{i: n}(x)=\theta g(x ; \xi)[1-G(x ; \xi)]^{-\gamma-1} e^{\frac{\theta}{\gamma}\left(1-\left[1-G\left(x_{i} ; \xi\right)\right]^{-\gamma}\right)} \sum_{j=0}^{\infty} \sum_{l=0}^{n-i} c_{j, l}\left(1-e^{\frac{\theta}{\gamma}\left(1-\left[1-G\left(x_{i} ; \xi\right)\right]^{-\gamma}\right)}\right)^{j+l+i-1} .
$$

By applying the generalized binomial series expansion

$$
\left(1-\left[1-G\left(x_{i} ; \xi\right)\right]^{-\gamma}\right)^{j+l+i-1}=\sum_{m=0}^{\infty}(-1)^{m}\left(\begin{array}{c}
j+l+i-1 \\
m
\end{array}\right) e^{\frac{\theta m}{\gamma}\left(1-\left[1-G\left(x_{i} ; \xi\right)\right]^{-\gamma}\right)},
$$

as well as equations (2.3), (2.4), and (2.5), we get

$$
\begin{aligned}
f_{i: n}(x)= & \sum_{j, m, n, p, q=0}^{\infty} \sum_{l=0}^{n-i} \frac{(-1)^{m+p} \theta^{n+1}(m+1)^{n}}{\gamma^{n} n !} C_{j, l}\left(\begin{array}{c}
j+l+i-1 \\
m
\end{array}\right)\left(\begin{array}{c}
n \\
p
\end{array}\right) \\
& \times\left(\begin{array}{c}
-\gamma(p+1)-1 \\
q
\end{array}\right) g(x ; \xi)[G(x ; \xi)]^{q}=\sum_{q=0}^{\infty} C_{q}^{*} g_{q}(x ; \xi),
\end{aligned}
$$

where $g_{q}(x ; \xi)=(q+1) g(x ; \xi)[G(x ; \xi)]^{q}$ is an Exp-G distribution with power parameter $q$ and

$$
C_{q}^{*}=\sum_{j, m, n, p=0}^{\infty} \sum_{l=0}^{n-i} \frac{(-1)^{m+p} \theta^{n+1}(m+1)^{n}}{(q+1) \gamma^{n} n !} C_{j, l}\left(\begin{array}{c}
j+l+i-1 \\
m
\end{array}\right)\left(\begin{array}{l}
n \\
p
\end{array}\right)\left(\begin{array}{c}
-\gamma(p+1)-1 \\
q
\end{array}\right) .
$$

\section{Estimation}

Let $X_{i} \sim M O-G o m-G(\gamma, \delta, \theta, \xi)$ with the parameter vector $\underline{\Psi}=(\gamma, \delta, \theta, \xi)^{\top}$, then the log-likelihood function $\ell=\ell(\underline{\Psi})$ from a random sample of size $n$ is given by

$$
\begin{aligned}
\ell(\underline{\Psi})= & n \log (2 \theta \delta)+\sum_{i=1}^{n} \log \left[g\left(x_{i} ; \xi\right)\right]-(\gamma+1) \sum_{i=1}^{n} \log \left[1-G\left(x_{i} ; \xi\right)\right] \\
& +\frac{\theta}{\gamma} \sum_{i=1}^{n}\left(1-\left[1-G\left(x_{i} ; \xi\right)\right]^{-\gamma}\right)-2 \sum_{i=1}^{n} \log \left[1-\bar{\delta} e^{\frac{\theta}{\gamma}\left(1-\left[1-G\left(x_{i} ; \xi\right)\right]^{-\gamma}\right)}\right] .
\end{aligned}
$$

The elements of the score vector $\mathbf{U}(\underline{\Psi})$ are given by

$$
\begin{aligned}
& \frac{\partial \ell}{\partial \delta}=\frac{n}{\delta}-2 \sum_{i=1}^{n} \frac{e^{\frac{\theta}{\gamma}\left(1-\left[1-G\left(x_{i} ; \xi\right)\right]^{-\gamma}\right)}}{1-\bar{\delta} e^{\frac{\theta}{\gamma}\left(1-\left[1-G\left(x_{i} ; \xi\right)\right]^{-\gamma}\right)}} \\
& \frac{\partial \ell}{\partial \theta}=\frac{n}{\theta}+\frac{1}{\gamma} \sum_{i=1}^{n}\left(1-\left[1-G\left(x_{i} ; \xi\right)\right]^{-\gamma}\right)+2 \sum_{i=1}^{n} \frac{\bar{\delta}\left(1-\left[1-G\left(x_{i} ; \xi\right)\right]-\gamma\right) e^{\frac{\theta}{\gamma}\left(1-\left[1-G\left(x_{i} ; \xi\right)\right]^{-\gamma}\right)}}{\gamma\left(1-\bar{\delta} e^{\frac{\theta}{\gamma}\left(1-\left[1-G\left(x_{i} ; \xi\right)\right]^{-\gamma}\right)}\right)} \\
& \frac{\partial \ell}{\partial \gamma}=\sum_{i=1}^{n} \theta \frac{\gamma\left(1-G\left(x_{i} ; \xi\right)\right)^{-\gamma} \log \left[1-G\left(x_{i} ; \xi\right)\right]-\left(1-\left(1-G\left(x_{i} ; \xi\right)\right)^{-\gamma}\right)}{\gamma^{2}}
\end{aligned}
$$




$$
\begin{aligned}
& +2 \sum_{i=1}^{n} \frac{\left(\gamma\left[1-G\left(x_{i} ; \xi\right)\right]^{-\gamma} \log \left[1-G\left(x_{i} ; \xi\right)\right]+\left(1-\left[1-G\left(x_{i} ; \xi\right)\right]^{-\gamma}\right)\right)}{\gamma^{2}\left(1-\bar{\delta} e^{\frac{\theta}{\gamma}\left(1-\left[1-G\left(x_{i} ; \xi\right)\right]^{-\gamma}\right)}\right)} \\
& \times \bar{\delta} \theta e^{\frac{\theta}{\gamma}\left(1-\left[1-G\left(x_{i} ; \xi\right)\right]^{-\gamma}\right)}-\sum_{i=1}^{n} \log \left[1-G\left(x_{i} ; \xi\right)\right] \\
\frac{\partial \ell}{\partial \xi_{k}}= & \sum_{i=1}^{n} \frac{\frac{\partial\left[g\left(x_{i} ; \xi\right)\right]}{\partial \xi_{k}}}{g\left(x_{i} ; \xi\right)}-(\gamma+1) \sum_{i=1}^{n} \frac{\frac{\partial\left[1-G\left(x_{i} ; \xi\right)\right]}{\partial \xi_{k}}}{\left[1-G\left(x_{i} ; \xi\right)\right]}+\theta \sum_{i=1}^{n} g\left(x_{i} ; \xi\right)\left(1-G\left(x_{i} ; \xi\right)\right)^{-\gamma-1} \\
& -2 \sum_{i=1}^{n} \frac{\frac{\partial\left[1-\bar{\delta} e^{\frac{\theta}{\gamma}\left(1-\left[1-G\left(x_{i} ; \xi\right)\right]^{-\gamma}\right)}\right.}{\partial \xi_{k}}}{\left[1-\bar{\delta} e^{\frac{\theta}{\gamma}\left(1-\left[1-G\left(x_{i} ; \xi\right)\right]^{-\gamma}\right)}\right]}
\end{aligned}
$$

respectively. We use software such as R, MATLAB to find numerical solutions of these partial derivatives for specified baseline cdf $G(x ; \xi)$. Furthermore, to obtain the estimates of the confidence intervals for the model parameters $\underline{\Psi}=(\gamma, \delta, \theta, \xi)$, we use the Fisher information matrix

$$
J(\underline{\Psi})=\left(\begin{array}{llll}
J_{\gamma \gamma}(\underline{\Psi}) & J_{\gamma \delta}(\underline{\Psi}) & J_{\gamma \delta}(\underline{\Psi}) & J_{\gamma \xi}(\underline{\Psi}) \\
J_{\delta \gamma}(\underline{\Psi}) & J_{\delta \delta}(\underline{\Psi}) & J_{\delta \theta}(\underline{\Psi}) & J_{\delta \xi}(\underline{\Psi}) \\
J_{\theta \gamma}(\underline{\Psi}) & J_{\theta \delta}(\underline{\Psi}) & J_{\theta \theta}(\underline{\Psi}) & J_{\theta \xi}(\underline{\Psi}) \\
J_{\xi \gamma}(\underline{\Psi}) & J_{\xi \delta}(\underline{\Psi}) & J_{\xi \theta}(\underline{\Psi}) & J_{\xi \xi}(\underline{\Psi})
\end{array}\right),
$$

where $J_{i, j}=\frac{-\partial^{2} \ell(\underline{\Psi})}{\partial i \partial j}$, for $i, j=(\gamma, \delta, \theta, \xi)$, where $\xi$ is a $p$ component vector, $J_{\xi \xi}(\underline{\Psi})$ is a $p \times p$ matrix, $\mathrm{J}_{\gamma \xi}(\underline{\Psi}), \mathrm{J}_{\delta \xi}(\underline{\Psi})$ and $\mathrm{J}_{\theta \xi}(\underline{\Psi})$ has $p \times 1$ components, respectively. Under the usual regularity conditions $\underline{\hat{\Psi}}$ is asymptotically normal distributed, that is $\underline{\hat{\Psi}} \sim \mathrm{N}\left(\underline{0}, \mathrm{I}^{-1}(\underline{\Psi})\right)$ as $\mathrm{n} \longrightarrow \infty$, where $\mathrm{I}(\underline{\Psi})$ is the expected information matrix. The asymptotic behavior remains valid if $\mathrm{I}(\underline{\Psi})$ is replaced by $J(\underline{\hat{\Psi}})$, the information matrix evaluated at $\underline{\hat{\underline{y}}}$.

\section{Some special cases}

In this section, we present some special cases for the MO-Gom-G family of distributions by considering the Weibull, Kumarswamy and Burr XII distributions as baseline distributions.

\subsection{Marshall-Olkin-Gompertz-Weibull (MO-Gom-W) distribution}

If we take the Weibull distribution as the baseline distribution with pdf and cdf given by $g(x ; \lambda)=$ $\lambda x^{\lambda-1} e^{-x^{\lambda}}$ and $G(x ; \lambda)=1-e^{-x^{\lambda}}$, respectively, for $\lambda>0$, we obtain the MO-Gom-W distribution with cdf, pdf and hrf given by

$$
\begin{aligned}
& F(x ; \theta, \gamma, \delta, \lambda)=\frac{1-e^{\frac{\theta}{\gamma}\left(1-\left[e^{-x^{\lambda}}\right]^{-\gamma}\right)}}{1-\bar{\delta}\left(e^{\frac{\theta}{\gamma}\left(1-\left[e^{\left.-x^{\lambda}\right]-\gamma}\right)\right.}\right.}, \\
& f(x ; \theta, \gamma, \delta, \lambda)=\frac{\delta \theta \lambda x^{\lambda-1} e^{-\chi^{\lambda}}\left[e^{-\chi^{\lambda}}\right]^{-\gamma-1} e^{\frac{\theta}{\gamma}\left(1-\left[e^{-x^{\lambda}}\right]^{-\gamma}\right)}}{\left(1-\bar{\delta}\left(e^{\frac{\theta}{\gamma}\left(1-\left[e^{-\chi^{\lambda}}\right]-\gamma\right.}\right)\right)^{2}} \\
& h(x ; \theta, \gamma, \delta, \lambda)=\frac{\delta \theta \lambda x^{\lambda-1} e^{-\chi^{\lambda}}\left[e^{-\chi^{\lambda}}\right]^{-\gamma-1} e^{\frac{\theta}{\gamma}\left(1-\left[e^{-\chi^{\lambda}}\right]^{-\gamma}\right)}}{\left(1-\bar{\delta}\left(e^{\frac{\theta}{\gamma}\left(1-\left[e^{-\chi^{\lambda}}\right]^{-\gamma}\right)}\right)\right)^{2}}\left[1-\frac{1-e^{\frac{\theta}{\gamma}\left(1-\left[e^{-\chi^{\lambda}}\right]^{-\gamma}\right)}}{1-\bar{\delta}\left(e^{\frac{\theta}{\gamma}\left(1-\left[e^{-\chi^{\lambda}}\right]-\gamma\right.}\right)}\right]^{-1},
\end{aligned}
$$

respectively, for $\theta, \gamma, \delta$ and $\lambda>0$.

Figure 1 shows plots of the pdfs and hrfs for the MO-Gom-W distribution. The pdf apply to heavy tailed data and with varying kurtosis. The hrf takes decreasing, increasing and bathtub shapes. 

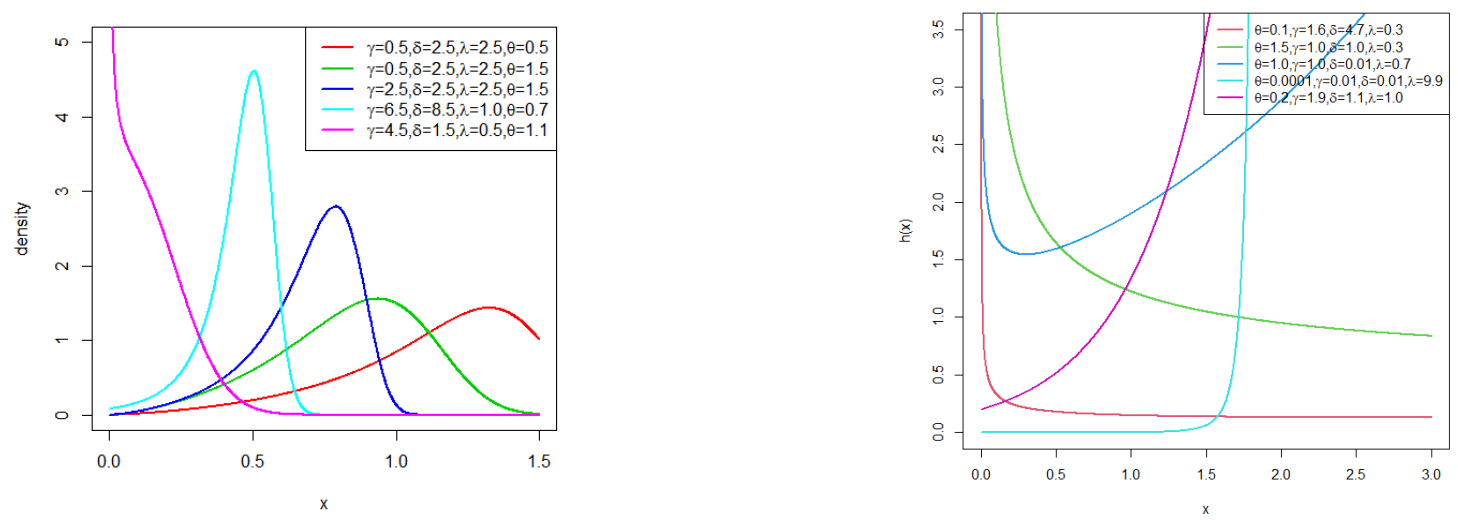

Figure 1: Plots of the pdf and hrf for the MO-Gom-W distribution.

\subsection{Marshall-Olkin-Gompertz-Kumaraswamy (MO-Gom-Kw) distribution}

By taking the baseline distribution to be a Kumaraswamy distribution with pdf and cdf given by $g(x ; a, b)=a b x^{a-1}\left(1-x^{a}\right)^{b-1}$ and $G(x ; a, b)=1-\left(1-x^{a}\right)^{b}$, respectively, for $a, b>0$, we obtain the MO-Gom-Kw distribution with cdf, pdf and hrf given by

$$
\begin{aligned}
& \mathrm{F}_{\mathrm{MO}-\mathrm{Gom}-\mathrm{G}}(x ; \theta, \gamma, \delta, \mathrm{a}, \mathrm{b})=\frac{1-e^{\frac{\theta}{\gamma}\left(1-\left[\left(1-\mathrm{x}^{\mathrm{a}}\right)^{\mathrm{b}}\right]^{-\gamma}\right)}}{1-\bar{\delta}\left(e^{\frac{\theta}{\gamma}\left(1-\left[\left(1-\mathrm{x}^{\mathrm{a}}\right)^{\mathrm{b}}\right]^{-\gamma}\right)}\right)}, \\
& f_{M O-G o m-G}(x ; \theta, \gamma, \delta, a, b)=\frac{\delta \theta a b x^{a-1}\left(1-x^{a}\right)^{b-1}\left[\left(1-x^{a}\right)^{b}\right]^{-\gamma-1} e^{\frac{\theta}{\gamma}\left(1-\left[\left(1-x^{a}\right)^{b}\right]^{-\gamma}\right)}}{\left(1-\bar{\delta}\left(e^{\frac{\theta}{\gamma}\left(1-\left[\left(1-x^{a}\right)^{b}\right]^{-\gamma}\right)}\right)\right)^{2}}, \\
& h(x ; \theta, \gamma, \delta, a, b)=\frac{\delta \theta a b x^{a-1}\left(1-x^{a}\right)^{b-1}\left[\left(1-x^{a}\right)^{b}\right]^{-\gamma-1} e^{\frac{\theta}{\gamma}\left(1-\left[\left(1-x^{a}\right)^{b}\right]^{-\gamma}\right)}}{\left(1-\bar{\delta}\left(e^{\frac{\theta}{\gamma}\left(1-\left[\left(1-\chi^{a}\right)^{b}\right]^{-\gamma}\right)}\right)\right)^{2}} \\
& \times\left[1-\frac{1-e^{\frac{\theta}{\gamma}\left(1-\left[\left(1-\chi^{a}\right)^{b}\right]^{-\gamma}\right)}}{1-\bar{\delta}\left(e^{\frac{\theta}{\gamma}\left(1-\left[\left(1-\chi^{a}\right)^{b}\right]^{-\gamma}\right)}\right)}\right]^{-1}
\end{aligned}
$$

respectively, for $\theta, \gamma, \delta, a, b>0$.
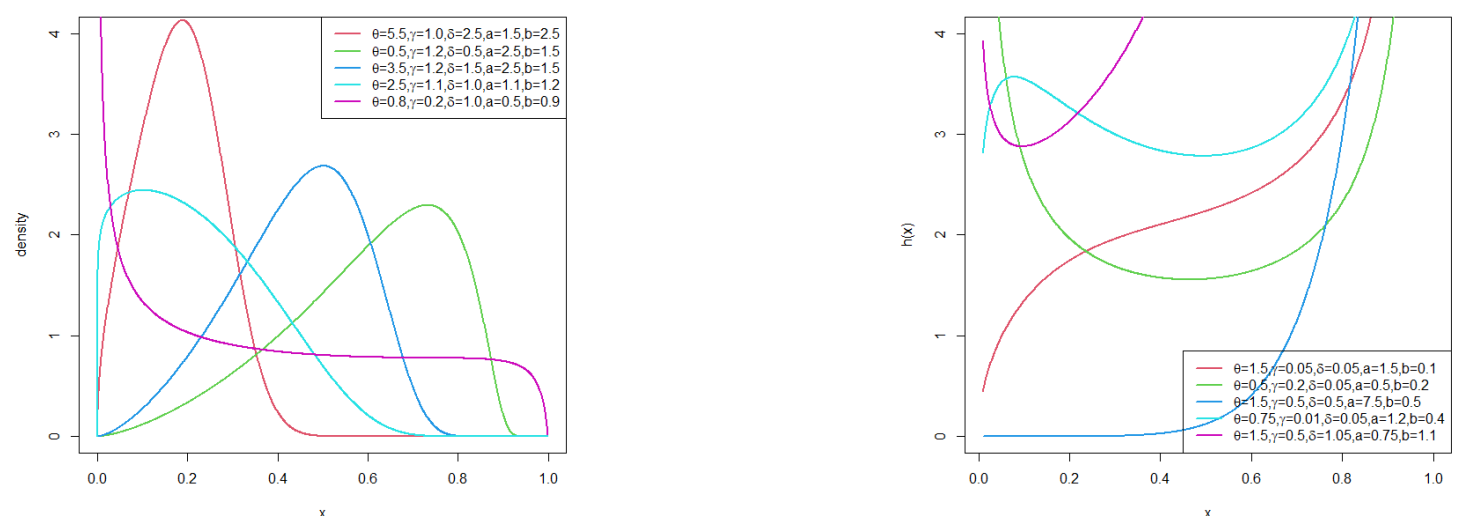

Figure 2: Plots of the pdf and hrf for the MO-Gom-Kw distribution.

Figure 2 shows plots of the pdfs and hrfs for the MO-Gom-Kw distribution. The pdf can apply to left 
or right-skewed data sets. The hrf exhibits increasing, bathtub and upside bathtub followed by bathtub shapes.

\subsection{Marshall-Olkin-Gompertz-Burr XII (MO-Gom-BXII) distribution}

If we consider the baseline distribution to be Burr XII distribution with pdf and cdf given by $g(x ; c, k)=$ $c k x^{c-1}\left(1+x^{c}\right)^{-k-1}$ and $G(x ; c, k)=1-\left(1+x^{c}\right)^{-k}$, respectively, we obtain the MO-Gom-BXII distribution with cdf, pdf, and hrf given by

$$
\begin{aligned}
& \mathrm{F}(x ; \theta, \gamma, \delta, c, k)=\frac{1-e^{\frac{\theta}{\gamma}\left(1-\left[\left(1+x^{c}\right)^{-k}\right]^{-\gamma}\right)}}{1-\bar{\delta}\left(e^{\frac{\theta}{\gamma}\left(1-\left[\left(1+x^{c}\right)^{-k}\right]^{-\gamma}\right)}\right)}, \\
& f(x ; \theta, \gamma, \delta, c, k)=\frac{\delta \theta c k x^{c-1}\left(1+x^{c}\right)^{-k-1}\left[\left(1+x^{c}\right)^{-k}\right]^{-\gamma-1} e^{\frac{\theta}{\gamma}\left(1-\left[\left(1+x^{c}\right)^{-k}\right]^{-\gamma}\right)}}{\left(1-\bar{\delta}\left(e^{\frac{\theta}{\gamma}\left(1-\left[\left(1+x^{c}\right)^{-k}\right]^{-\gamma}\right)}\right)\right)^{2}}, \\
& h(x ; \theta, \gamma, \delta, c, k)=\frac{\left.\delta \theta c k x^{c-1}\left(1+x^{c}\right)^{-k-1}\left[\left(1+x^{c}\right)^{-k}\right]^{-\gamma-1} e^{\frac{\theta}{\gamma}\left(1-\left[\left(1+x^{c}\right)^{-k}\right]-\gamma\right.}\right)}{\left(1-\bar{\delta}\left(e^{\frac{\theta}{\gamma}\left(1-\left[\left(1+x^{c}\right)^{-k}\right]^{-\gamma}\right)}\right)\right)^{2}} \\
& \times\left[1-\frac{1-e^{\frac{\theta}{\gamma}\left(1-\left[\left(1+x^{c}\right)^{-k}\right]^{-\gamma}\right)}}{1-\bar{\delta}\left(e^{\frac{\theta}{\gamma}\left(1-\left[\left(1+x^{c}\right)^{-k}\right]^{-\gamma}\right)}\right)}\right]^{-1},
\end{aligned}
$$

respectively, for $\theta, \gamma, \delta, c, k>0$. We obtain the MO-Gom-Log-Logistic (MO-Gom-LLoG) and MO-GomLomax (MO-Gom-Lx) from the MO-Gom-BXII distribution by setting $k=1$ and $c=1$, respectively.
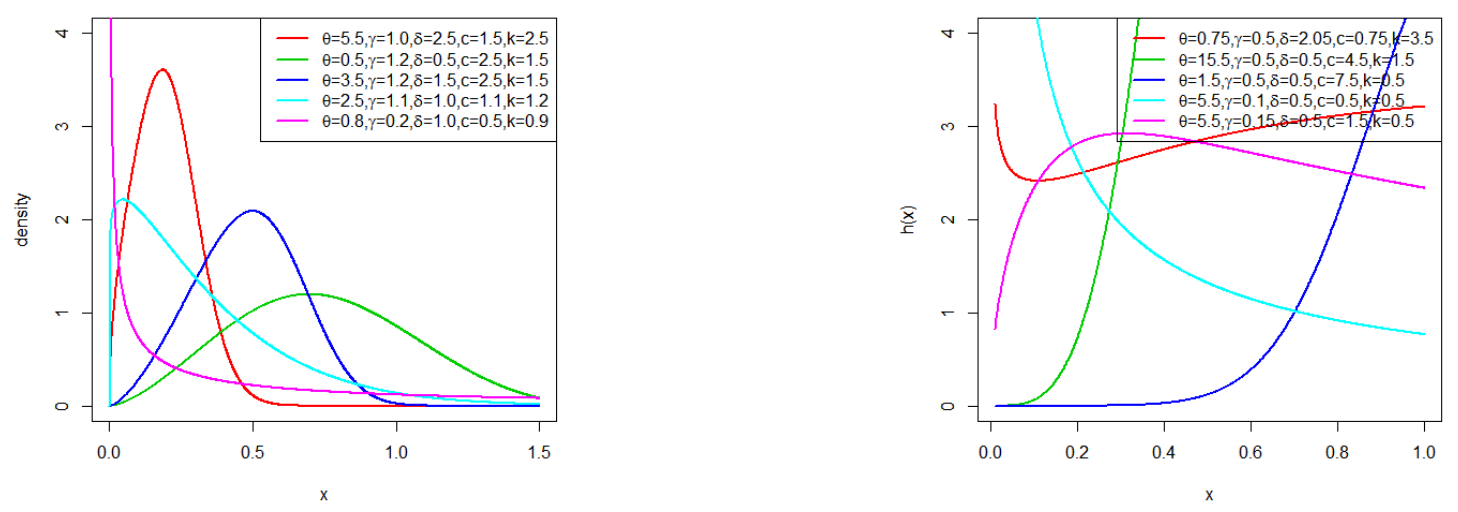

Figure 3: Plots of the pdf and hrf for the MO-Gom-BXII distribution.

Pdfs and hrfs plots for the MO-Gom-BXII distribution are shown in Figure 3. The pdf applies to heavy tailed data sets. The hrf exhibits decreasing, increasing, bathtub and upside bathtub shapes.

\section{Simulation study}

In this section, we present results of simulation study for the MO-Gom-W distribution. We fix the parameter $\theta=1$ in the following sets of parameter values (I: $\gamma=0.05, \delta=1.0, \lambda=0.5),($ II: $\gamma=1.0, \delta=$ $0.5, \lambda=0.01)$, (III: $\gamma=0.01, \delta=1.1, \lambda=0.5),(\mathrm{IV}: \gamma=0.01, \delta=1.0, \lambda=0.01),(\mathrm{V}: \gamma=1.0, \delta=0.5, \lambda=0.01)$, and (VI: $\gamma=0.01, \delta=1.1, \lambda=1.1$ ). We consider sample sizes $n=25,50,100,200,400$ and 800, for $N=1000$ for each sample. We estimate the mean, average bias and root mean square error (RMSE). The bias and RMSE for the estimated parameter, say, $\hat{\Delta}$, say, are given by:

$$
\operatorname{Bias}(\hat{\Delta})=\frac{1}{N} \sum_{i=1}^{N}\left(\hat{\Delta}_{i}-\Delta\right), \quad \text { and } \operatorname{RMSE}(\hat{\Delta})=\sqrt{\frac{\sum_{i=1}^{N}\left(\hat{\Delta}_{i}-\Delta\right)^{2}}{N}}
$$


respectively. From the results in Table 1, the mean values approximate the true parameter values, RMSE and bias decay towards zero for all the parameter values. We therefore, conclude that our model give consistent maximum likelihood estimates (MLEs).

Table 1: Monte Carlo simulation results for MO-Gom-W distribution: mean, RMSE, and average bias

\begin{tabular}{|c|c|c|c|c|c|c|c|c|c|c|}
\hline & & \multicolumn{3}{|c|}{ I: $\gamma=0.05, \delta=1.0, \lambda=0.5$} & \multicolumn{3}{|c|}{ III $\gamma=1.0, \delta=0.5, \lambda=0.01$} & \multicolumn{3}{|c|}{$\overline{~ I I I: ~} \gamma=0.01, \delta=1.1, \lambda=0.5$} \\
\hline & $\mathrm{n}$ & Mean & RMSE & Bias & Mean & RMSE & Bias & Mean & RMSE & Bias \\
\hline & 25 & 0.089236 & 0.083568 & 0.039236 & 1.838723 & 4.276864 & 0.838723 & 0.016832 & 0.021484 & 0.006832 \\
\hline & 50 & 0.065115 & 0.038042 & 0.015115 & 1.382312 & 2.389007 & 0.382312 & 0.012038 & 0.008697 & 0.002038 \\
\hline \multirow[t]{6}{*}{$\gamma$} & 100 & 0.056817 & 0.022569 & 0.006817 & 1.148946 & 0.282355 & 0.148946 & 0.010859 & 0.004961 & 0.000859 \\
\hline & 200 & 0.053659 & 0.015359 & 0.003659 & 1.077162 & 0.172782 & 0.077162 & 0.010629 & 0.003327 & 0.000629 \\
\hline & 400 & 0.051052 & 0.009684 & 0.001052 & 1.050306 & 0.102636 & 0.050306 & 0.010233 & 0.002036 & 0.000233 \\
\hline & 800 & 0.050612 & 0.007122 & 0.000612 & 0.980394 & 0.019606 & -0.019606 & 0.010135 & 0.001351 & 0.000135 \\
\hline & 25 & 0.950591 & 0.190381 & -0.049409 & 0.426400 & 0.115719 & -0.073600 & 1.117623 & 0.171134 & 0.017623 \\
\hline & 50 & 0.979546 & 0.130185 & -0.020454 & 0.434876 & 0.087382 & -0.065124 & 1.104701 & 0.117455 & 0.004701 \\
\hline \multirow[t]{6}{*}{$\varepsilon$} & 100 & 0.993832 & 0.097661 & -0.006168 & 0.442264 & 0.067449 & -0.057736 & 1.105440 & 0.087184 & 0.005440 \\
\hline & 200 & 0.998313 & 0.074440 & -0.001687 & 0.460930 & 0.046620 & -0.039070 & 1.100406 & 0.064267 & 0.000406 \\
\hline & 400 & 1.003138 & 0.052508 & 0.003138 & 0.471023 & 0.031762 & -0.028977 & 1.101555 & 0.045123 & 0.001555 \\
\hline & 800 & 1.000015 & 0.037477 & 0.000015 & 0.479790 & 0.020210 & -0.020210 & 1.099557 & 0.028943 & -0.000443 \\
\hline & 25 & 1.861678 & 2.170055 & 1.361678 & 0.393966 & 0.506445 & 0.383966 & 1.248728 & 2.215944 & 0.748728 \\
\hline & 50 & 1.203697 & 1.234740 & 0.703697 & 0.195961 & 0.302119 & 0.185961 & 0.995741 & 1.744226 & 0.495741 \\
\hline \multirow[t]{7}{*}{$\lambda$} & 100 & 0.881350 & 0.730413 & 0.381350 & 0.134571 & 0.159950 & 0.124571 & 0.735833 & 1.112693 & 0.235833 \\
\hline & 200 & 0.723582 & 0.523697 & 0.223582 & 0.084699 & 0.095748 & 0.074699 & 0.671994 & 0.777442 & 0.171994 \\
\hline & 400 & 0.592761 & 0.292004 & 0.092761 & 0.056989 & 0.058520 & 0.046989 & 0.587842 & 0.382424 & 0.087842 \\
\hline & 800 & 0.553761 & 0.198919 & 0.053761 & 0.034180 & 0.024180 & 0.024180 & 0.558582 & 0.265177 & 0.058582 \\
\hline & & \multicolumn{3}{|c|}{ IV: $\gamma=0.01, \delta=1.0, \lambda=0.01$} & \multicolumn{3}{|c|}{$\mathrm{V}: \gamma=1.1, \delta=0.5, \lambda=0.01$} & \multicolumn{3}{|c|}{ VI: $\gamma=0.01, \delta=1.1, \lambda=1.1$} \\
\hline & 25 & 0.016035 & 0.018195 & 0.006035 & 1.857379 & 3.721958 & 0.757379 & 0.018120 & 0.026549 & 0.008120 \\
\hline & 50 & 0.011973 & 0.006729 & 0.001973 & 1.551204 & 2.289800 & 0.451204 & 0.011984 & 0.007663 & 0.001984 \\
\hline \multirow[t]{6}{*}{$\gamma$} & 100 & 0.011149 & 0.004883 & 0.001149 & 1.262386 & 0.327595 & 0.162386 & 0.010940 & 0.005038 & 0.000940 \\
\hline & 200 & 0.010508 & 0.002870 & 0.000508 & 1.205009 & 0.198097 & 0.105009 & 0.010503 & 0.003388 & 0.000503 \\
\hline & 400 & 0.010109 & 0.001848 & 0.000109 & 1.154345 & 0.113701 & 0.054345 & 0.010171 & 0.002038 & 0.000171 \\
\hline & 800 & 0.009844 & 0.001218 & -0.000156 & 1.074254 & 0.025746 & -0.025746 & 0.010198 & 0.001346 & 0.000198 \\
\hline & 25 & 1.029543 & 0.156270 & 0.029543 & 0.419907 & 0.116164 & -0.080093 & 1.110620 & 0.175366 & 0.010620 \\
\hline & 50 & 1.028252 & 0.104676 & 0.028252 & 0.428007 & 0.092837 & -0.071993 & 1.106311 & 0.117973 & 0.006311 \\
\hline \multirow[t]{6}{*}{$\delta$} & 100 & 1.022663 & 0.081232 & 0.022663 & 0.440975 & 0.069665 & -0.059025 & 1.105149 & 0.087988 & 0.005149 \\
\hline & 200 & 1.007736 & 0.058596 & 0.007736 & 0.458969 & 0.048402 & -0.041031 & 1.103304 & 0.066246 & 0.003304 \\
\hline & 400 & 1.008353 & 0.041202 & 0.008353 & 0.474596 & 0.029180 & -0.025404 & 1.102942 & 0.045146 & 0.002942 \\
\hline & 800 & 1.007543 & 0.029835 & 0.007543 & 0.480051 & 0.019949 & -0.019949 & 1.097270 & 0.027303 & -0.002730 \\
\hline & 25 & 0.695910 & 2.067498 & 0.685910 & 0.383818 & 0.484616 & 0.373818 & 1.721757 & 2.093511 & 0.621757 \\
\hline & 50 & 0.481176 & 1.607680 & 0.471176 & 0.202516 & 0.320988 & 0.192516 & 1.593124 & 1.885741 & 0.493124 \\
\hline \multirow[t]{4}{*}{$\lambda$} & 100 & 0.351372 & 1.118785 & 0.341372 & 0.132733 & 0.162065 & 0.122733 & 1.340366 & 1.140527 & 0.240366 \\
\hline & 200 & 0.177597 & 0.592742 & 0.167597 & 0.084059 & 0.093471 & 0.074059 & 1.225963 & 0.694144 & 0.125963 \\
\hline & 400 & 0.093874 & 0.328087 & 0.083874 & 0.047752 & 0.050881 & 0.037752 & 1.180181 & 0.401081 & 0.080181 \\
\hline & 800 & 0.061872 & 0.179336 & 0.051872 & 0.032694 & 0.022694 & 0.022694 & 1.142537 & 0.277465 & 0.042537 \\
\hline
\end{tabular}

\section{Applications}

In this section, we present two real data examples to demonstrate the flexibility of the MO-Gom$\mathrm{W}$ distribution in data fitting. The maximum likelihood estimation technique is used to estimate the model parameters via the nlm package in R software [28]. The AdequacyModel package in R software [28] is used to assess model performance. We consider the following goodness-of-fit statistics; Cramervon-Mises $\left(W^{*}\right)$ and Andersen-Darling $\left(A^{*}\right)$, as well as -2loglikelihood (-2 log L), Akaike Information Criterion (AIC), Consistent Akaike Information Criterion (AICC), Bayesian Information Criterion (BIC), Kolmogorov-Smirnov (K-S) statistic (and it's p-value), and sum of squares (SS). The model with the smallest values of the goodness-of-fit statistics and a bigger p-value for the K-S statistic is regarded as the best model.

We present data analysis results in Tables 2 and 3, parameter estimates (standard errors in parentheses) and the goodness-of-fit statistics for the various models considered in this paper. We also provide fitted densities and probability plots (as described by Chambers et al. [7]) to demonstrate the flexibility of the MO-Gom-W distribution in data fitting compared to the selected competing models (see Figures 4 and 5, for details).

We compare the MO-Gom-W distribution to the following models: Gompertz distribution by Gompertz [19], generalized Gompertz (G-Gom) distribution by El-Gohary at al. [16], Marshall-Olkin loglogistic (MO-LLoG) distribution by Wenhao [20], Marshall-Olkin extended inverse Weibull (MO-IW) by 
Pakungwati et al. [27], Marshall-Olkin extended Weibull (MO-EW) by Barreto-Souza and Baukoch [4], exponentiated half logistic odd Weibull-Topp-Leone-log logistic (EHLOW-TL-LLoG) by Chipepa et al. [11], odd generalized half logistic Weibull-Weibull (OGHLW-W) by Chipepa et al. [8], Kumaraswamy Weibull (KwW) by Cordeiro et al. [15], the exponential Lindley odd log-logistic Weibull (ELOLLW) by Korkmaz et al. [25], Kumaraswamy odd Lindley-log logistic (KOL-LLoG) by Chipepa et al. [10] and beta odd Lindley-exponential (BOL-E) by Chipepa et al. [9]. The pdfs of the non-nested models are

$$
f_{G-G o m}(x ; \alpha, \beta, \lambda)=\alpha \beta e^{\lambda x}\left(1-e^{\frac{-\beta}{\lambda}\left(e^{\lambda x}-1\right)}\right)^{\alpha-1},
$$

for $\alpha, \beta, \lambda>0$,

$$
\mathrm{f}_{\mathrm{MO}-\mathrm{EW}}(x ; \alpha, \gamma, \lambda)=\frac{\alpha \gamma \lambda^{\gamma} \chi^{\gamma-1} e^{-\lambda x^{\gamma}}}{\left(1-\bar{\alpha} e^{-\lambda x^{\omega}}\right)^{2}}
$$

for $\alpha, \gamma, \lambda>0$,

$$
f_{\mathrm{MO}-\mathrm{IW}}(x ; \alpha, \theta, \lambda)=\frac{\alpha \lambda \theta^{-\lambda} x^{-\lambda-1} e^{-(\theta x)^{-\lambda}}}{\left[\alpha-(\alpha-1) e^{\left.-(\theta x)^{-\lambda}\right]^{2}}\right.}
$$

for $\alpha, \theta, \lambda>0$,

$$
f_{\mathrm{MO}-\mathrm{LLOG}}(x ; \alpha, \beta, \gamma)=\frac{\alpha^{\beta} \beta \gamma x^{\beta-1}}{\left(x^{\beta}+\alpha^{\beta} \gamma\right)^{2}}
$$

for $\alpha, \beta, \gamma>0$,

$$
\begin{aligned}
f_{\text {EHLOW-TL-BXII }}(x ; \alpha, \beta, \delta, \lambda, \gamma)= & \frac{4 \alpha \beta \delta \lambda \gamma x^{\lambda-1}\left(1+x^{\lambda}\right)^{-2 \gamma-1}\left[1-\left(1+x^{\lambda}\right)^{-2 \gamma}\right]^{\alpha \beta-1}}{\left(1-\left[1-\left(1+x^{\lambda}\right)^{-2 \gamma}\right]^{\alpha}\right)^{\beta+1}} \\
& \times \exp (-t)(1+\exp (-t))^{-2}\left[\frac{1-\exp (-t)}{1+\exp (-t)}\right]^{\delta-1},
\end{aligned}
$$

where $t=\left[\frac{\left[1-\left(1+x^{\lambda}\right)^{-2 \gamma}\right]^{\alpha}}{1-\left[1-\left(1+x^{\lambda}\right)^{-2 \gamma}\right]^{\alpha}}\right]^{\beta}$, for $\alpha, \beta, \delta, \lambda, \gamma>0$,

$$
f_{\text {OGHLW-W }}(x ; \alpha, \beta, \lambda, \gamma)=\frac{2 \alpha \beta \lambda \gamma x^{\gamma-1} e^{-\lambda x \gamma}\left(1-e^{-\lambda x \gamma}\right)^{\beta-1} \exp \left\{-\alpha\left[\frac{1-e^{-\lambda x \gamma}}{e^{-\lambda x \gamma}}\right]^{\beta}\right\}}{e^{-(\beta+1) \lambda x \gamma}\left(1+\exp \left\{-\alpha\left[\frac{1-e^{-\lambda x \gamma}}{e^{-\lambda x \gamma}}\right]^{\beta}\right\}\right)^{2}},
$$

for $\alpha, \beta, \lambda, \gamma>0$,

$$
f_{K w W}(x ; a, b, \alpha, \beta)=a b \alpha^{\beta} x^{\beta-1} e^{-(\alpha x)^{\beta}}\left(1-e^{-(\alpha x)^{\beta}}\right)^{a-1}\left(1-\left(1-e^{-(\alpha x)^{\beta}}\right)^{a}\right)^{b-1},
$$

for $a, b, \alpha, \beta>0$,

$$
\begin{aligned}
& f_{\text {ELOLLW }}(x ; \alpha, \beta, \gamma, \theta, \lambda)=\frac{\alpha \theta^{2} \gamma \lambda^{\gamma} x^{\gamma-1} e^{-(\lambda x)^{\gamma}}\left(e^{-(\lambda x)}\right)^{\alpha \theta-1}\left(1-e^{-(\lambda x)^{\gamma}}\right)^{\alpha-1}}{(\theta+\beta)\left(\left(1-e^{-(\lambda x)^{\gamma}}\right)^{\alpha}+e^{-\alpha(\lambda x)^{\gamma}}\right)^{\theta-1}} \\
& \times\left(1-\beta \log \left[\frac{e^{-(\lambda x)^{\gamma}}}{\left(1-e^{-(\lambda x)^{\gamma}}\right)^{\alpha}+e^{-\alpha(\lambda x)^{\gamma}}}\right]\right),
\end{aligned}
$$

for $\alpha, \beta, \gamma, \theta, \lambda>0$,

$$
\begin{aligned}
f_{\text {KOL-LLoG }}(x ; a, b, \lambda, c)= & a b\left[\frac{\lambda^{2}}{(1+\lambda)} \frac{c x^{c}-1}{\left(1+x^{c}\right)^{-1}} \exp (-\lambda z)\right]\left[1-\frac{\lambda+\left(\left(1+x^{c}\right)^{-1}\right)}{(1+\lambda)\left(\left(1+x^{c}\right)^{-1}\right)} \exp (-\lambda z)\right]^{a-1} \\
& \times\left(1-\left[1-\frac{\lambda+\left(\left(1+x^{c}\right)^{-1}\right)}{(1+\lambda)\left(\left(1+x^{c}\right)^{-1}\right)} \exp (-\lambda z)\right]^{a}\right)^{b-1}
\end{aligned}
$$

where $z=\frac{\left(1-\left(1+x^{c}\right)^{-1}\right)}{\left(\left(1+x^{c}\right)^{-1}\right)}, a, b, \lambda, c>0$, and

$$
f_{B O L-E}(x ; a, b, \lambda, \theta)=\frac{1}{B(a, b)}\left[1-\frac{\lambda+e^{-\theta x}}{(1+\lambda) e^{-\theta x}} \exp \left\{-\lambda \frac{\left(1-e^{-\theta x}\right)}{e^{-\theta x}}\right\}\right]^{a-1}
$$




$$
\times\left[\frac{\lambda+e^{-\theta x}}{(1+\lambda) e^{-\theta x}} \exp \left\{-\lambda \frac{\left(1-e^{-\theta x}\right)}{e^{-\theta x}}\right\}\right]^{b-1} \frac{\lambda^{2}}{(1+\lambda)} \frac{\left(\theta e^{-\theta x}\right)}{e^{-3 \theta x}} \exp \left\{-\lambda \frac{1-e^{-\theta x}}{e^{-\theta x}}\right\}
$$

for $a, b, \lambda, \theta>0$. We get the Gompertz distribution from G-Gom distribution by setting $\alpha=1$. Also, we obtain the EHLOW-TL-LLoG distribution from the EHLOW-TL-BXII distribution by setting $\gamma=1$. For the ELOLLOW distribution we considered the case when $\alpha=1$.

\subsection{Strengths of $1.5 \mathrm{~cm}$ glass fibres data}

The first data set represents strengths of $1.5 \mathrm{~cm}$ glass fibres. The data set was also analyzed by Bourguignon et al. [6] and Smith and Naylor [33]. The data are 0.55, 0.93, 1.25, 1.36, 1.49, 1.52, 1.58, 1.61, 1.64, 1.68, 1.73, 1.81, 2.00, $0.74,1.04,1.27,1.39,1.49,1.53,1.59,1.61,1.66,1.68,1.76,1.82,2.01,0.77,1.11,1.28,1.42,1.50,1.54,1.60,1.62,1.66$, $1.69,1.76,1.84,2.24,0.81,1.13,1.29,1.48,1.50,1.55,1.61,1.62,1.66,1.70,1.77,1.84,0.84,1.24,1.30,1.48,1.51,1.55$, $1.61,1.63,1.67,1.70,1.78,1.89$.

The estimated variance-covariance matrix for the MO-Gom-W model based on glass fibres data set is

$$
\left[\begin{array}{cccc}
1.0655 \times 10^{-6} & 8.8229 \times 10^{-8} & 3.2278 \times 10^{-5} & -3.1485 \times 10^{-7} \\
8.8229 \times 10^{-8} & 7.3053 \times 10^{-9} & 2.6726 \times 10^{-6} & -2.6071 \times 10^{-8} \\
3.2278 \times 10^{-5} & 2.6726 \times 10^{-6} & 9.7776 \times 10^{-4} & -9.5380 \times 10^{-6} \\
-3.1485 \times 10^{-7} & -2.6071 \times 10^{-8} & -9.5380 \times 10^{-6} & 1.0402 \times 10^{-7}
\end{array}\right]
$$

and the $95 \%$ confidence intervals for the model parameters are given by $\gamma \in[8.3180 \pm 0.40020], \delta \in[21.4720 \pm 0.0002]$, $\lambda \in[0.3134 \pm 0.0613]$ and $\theta \in[0.0018 \pm 0.0006]$. We observe from the results presented in Table 2 that the MO-Gom-

Table 2: MLEs and goodness-of-fit statistics

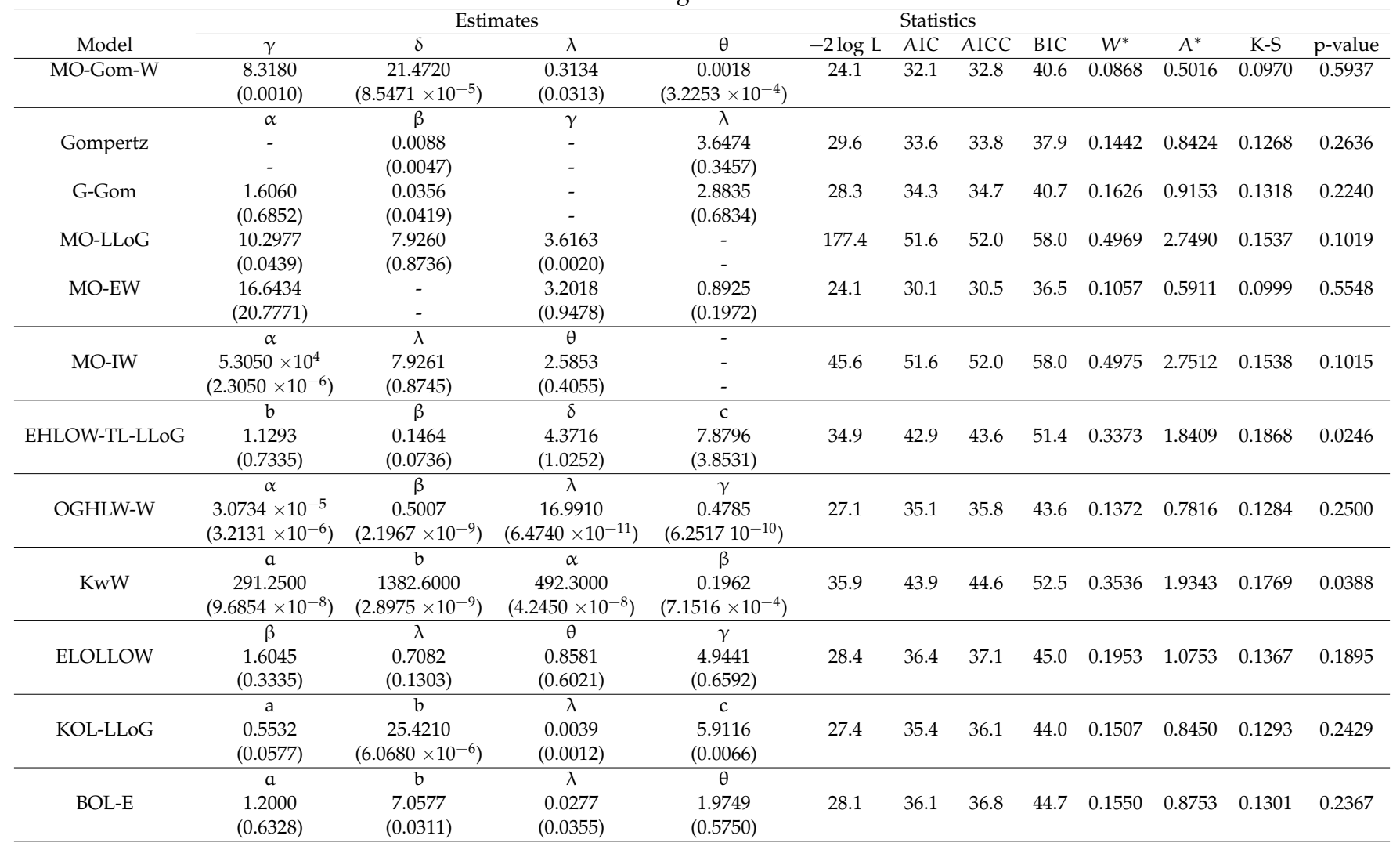

W distribution fits the glass fibres data set better than the other models since it has the lowest values for the goodness-of-fit statistics $A^{*}, W^{*}$ and K-S (and the largest p-value for the K-S statistic). Also, from fitted densities and probability plots shown in Figure 4, we observe that the MO-Gom-W model fit the data set better than the other models that were considered. 

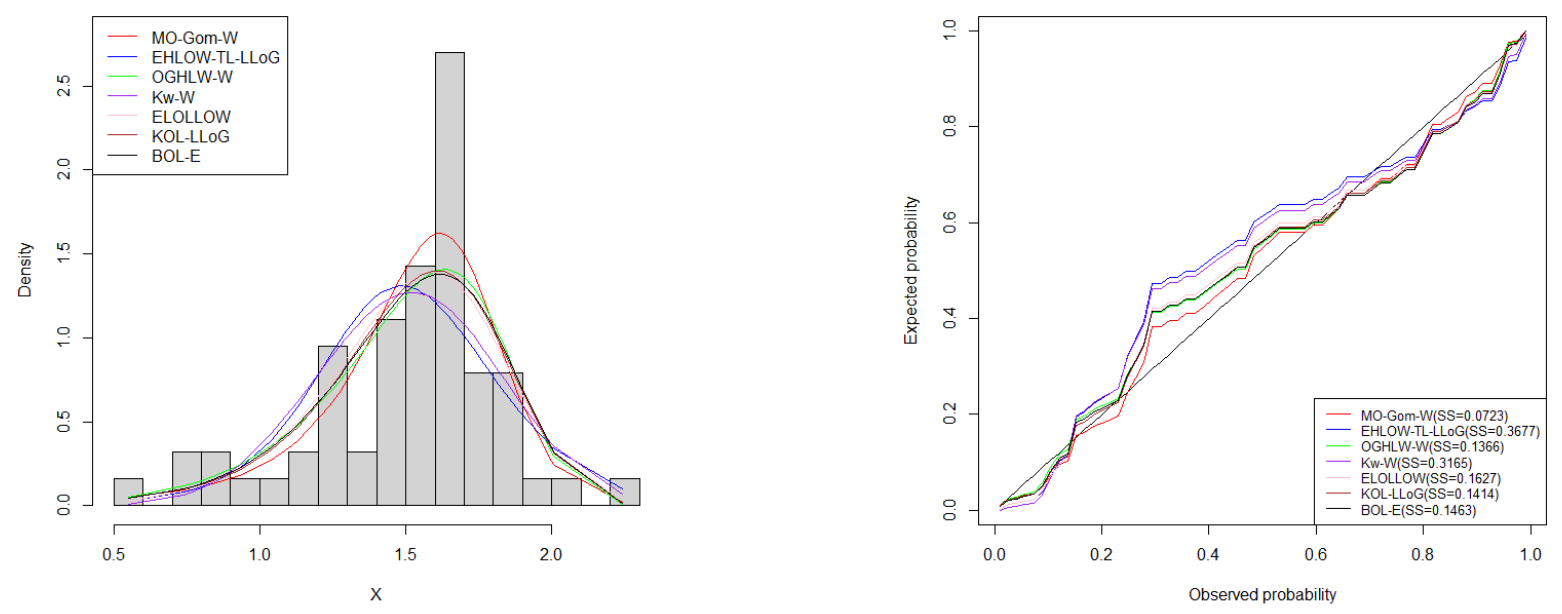

Figure 4: Fitted pdfs and probability plots for glass fibres data set.

\subsection{Turbocharger failure times data}

The data represents failure times $\left(10^{3} \mathrm{~h}\right)$ of turbocharger of one type of engine as report by $\mathrm{Xu}$ et al [35]. The data is $1.6,2.0,2.6,3.0,3.5,3.9,4.5,4.6,4.8,5.0,5.1,5.3,5.4,5.6,5.8,6.0,6.0,6.1,6.3,6.5,6.5,6.7,7.0,7.1,7.3,7.3,7.3$, 7.7, 7.7, 7.8, 7.9, 8.0, 8.1, 8.3, 8.4, 8.4, 8.5, 8.7, 8.8, 9.0.

The estimated variance-covariance matrix is

$$
\left[\begin{array}{cccc}
0.0018 & 0.0058 & -0.0384 & 1.5976 \times 10^{-4} \\
0.0058 & 1.0817 & -0.3088 & 5.6079 \times 10^{-3} \\
-0.0384 & -0.3088 & 0.8593 & -4.3962 \times 10^{-3} \\
0.0001 & 0.0056 & -0.0043 & 4.0375 \times 10^{-5}
\end{array}\right]
$$

and the $95 \%$ confidence intervals for the model parameters are given by $\gamma \in[0.0181 \pm 0.0832], \delta \in[0.7477 \pm 2.0386]$, $\lambda \in[2.3312 \pm 1.8169]$ and $\theta \in[0.0030 \pm 0.0125]$.
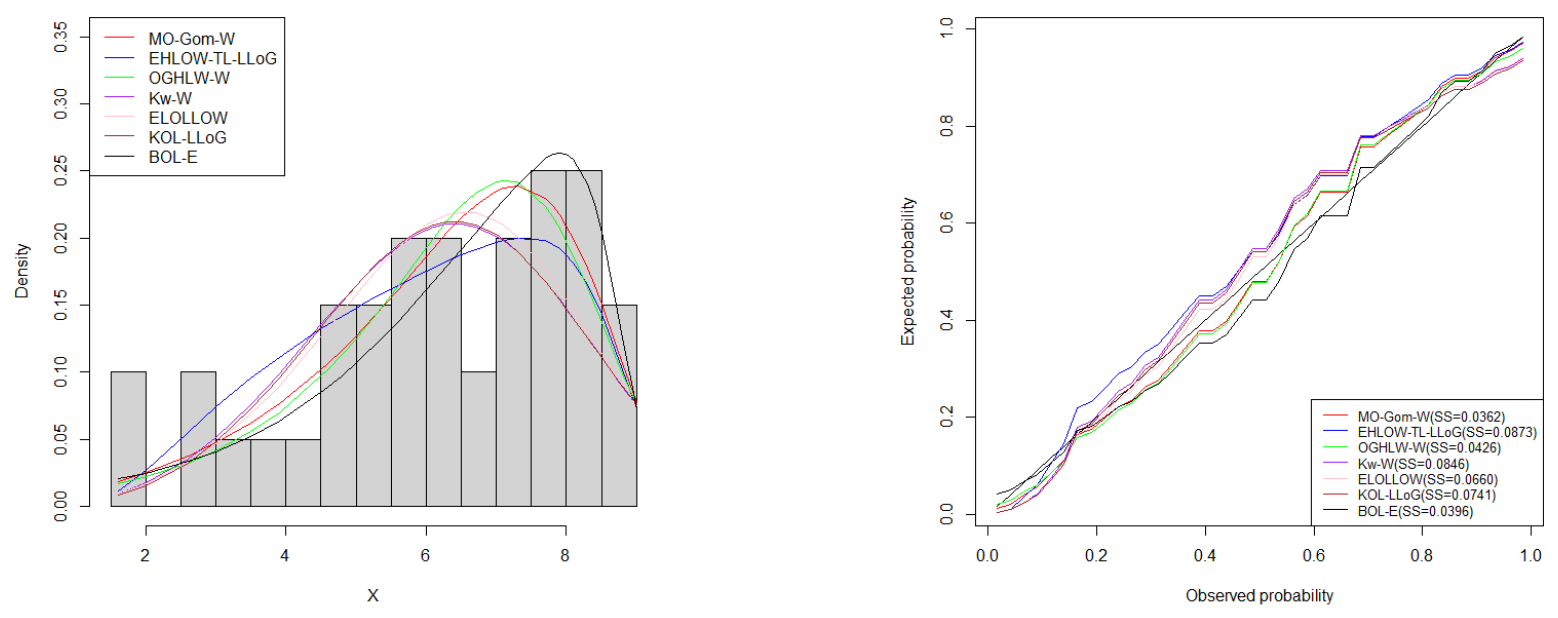

Figure 5: Fitted pdfs and probability plots for turbocharger data set.

Furthermore, results from the second example shown in Table 3 affirm that the MO-Gom-W model performs better than the other models considered since it has the lowest values for the goodness-of-fit statistics $A^{*}, W^{*}$ and $\mathrm{K}-\mathrm{S}$ (and the largest p-value for the K-S statistic). Figure 5 shows the flexibility gained by using the MO-Gom-W model on turbocharger data set compared to the other models that were considered. 
Table 3: MLEs and goodness-of-fit statistics.

\begin{tabular}{|c|c|c|c|c|c|c|c|c|c|c|c|c|}
\hline \multirow[b]{2}{*}{ Model } & \multicolumn{4}{|c|}{ Estimates } & \multicolumn{4}{|c|}{ Statistics } & \multirow[b]{2}{*}{$W^{*}$} & \multirow[b]{2}{*}{$A^{*}$} & \multirow[b]{2}{*}{ K-S } & \multirow[b]{2}{*}{$\mathrm{p}$-value } \\
\hline & $\gamma$ & $\delta$ & $\lambda$ & $\theta$ & $-2 \log \mathrm{L}$ & AIC & AICC & BIC & & & & \\
\hline MO-Gom-W & $\begin{array}{c}0.0181 \\
(0.0424)\end{array}$ & $\begin{array}{c}0.7477 \\
(1.0401)\end{array}$ & $\begin{array}{c}2.3312 \\
(0.9270)\end{array}$ & $\begin{array}{c}0.0030 \\
(0.0064)\end{array}$ & 158.3 & 166.3 & 167.4 & 173.0 & 0.0230 & 0.1704 & 0.0821 & 0.9501 \\
\hline Gompertz & $\begin{array}{l}\alpha \\
- \\
-\end{array}$ & $\begin{array}{c}\beta \\
0.4908 \\
(0.0914)\end{array}$ & $\begin{array}{l}\gamma \\
- \\
-\end{array}$ & $\begin{array}{c}\lambda \\
3.7553 \times 10^{-10} \\
(0.0094)\end{array}$ & 302.4 & 306.4 & 306.7 & 309.8 & 0.1896 & 1.2707 & 0.7401 & $<2.2000 \times 10^{-16}$ \\
\hline G-Gom & $\begin{array}{c}9.5146 \\
(5.2499)\end{array}$ & $\begin{array}{c}0.4498 \\
(0.2038)\end{array}$ & - & $\begin{array}{c}2.9039 \times 10^{-6} \\
(0.035)\end{array}$ & 180.3 & 186.3 & 187.0 & 191.4 & 0.2757 & 1.7601 & 0.1542 & 0.2976 \\
\hline MO-LLoG & $\begin{array}{c}4.6878 \\
(0.3149)\end{array}$ & $\begin{array}{c}4.8416 \\
(0.6536)\end{array}$ & $\begin{array}{c}3.9474 \\
(0.0772)\end{array}$ & $\begin{array}{l}- \\
-\end{array}$ & 177.4 & 183.4 & 184.1 & 188.5 & 0.2142 & 1.4072 & 0.1437 & 0.3807 \\
\hline MO-EW & $\begin{array}{c}4.8588 \\
(5.6658)\end{array}$ & $\begin{array}{l}- \\
-\end{array}$ & $\begin{array}{c}2.7877 \\
(0.8728)\end{array}$ & $\begin{array}{c}0.1887 \\
(0.0468)\end{array}$ & 162.6 & 168.6 & 169.3 & 173.7 & 0.0496 & 0.3766 & 0.0918 & 0.8889 \\
\hline MO-IW & $\begin{array}{c}\alpha \\
4.8362 \times 10^{3} \\
\left(8.4147 \times 10^{-6}\right) \\
\end{array}$ & $\begin{array}{c}\lambda \\
4.8391 \\
(0.6512) \\
\end{array}$ & $\begin{array}{c}\theta \\
0.9275 \\
(0.2303) \\
\end{array}$ & $\begin{array}{l}- \\
- \\
- \\
\end{array}$ & 177.4 & 183.4 & 184.1 & 188.5 & 0.2153 & 1.4131 & 0.1438 & 0.3800 \\
\hline EHLOW-TL-LLoG & $\begin{array}{c}\mathrm{b} \\
29.7753 \\
(13.9088)\end{array}$ & $\begin{array}{c}\beta \\
4.7226 \\
(2.5254)\end{array}$ & $\begin{array}{c}\delta \\
0.2243 \\
(0.1387)\end{array}$ & $\begin{array}{c}c \\
0.8263 \\
(0.1261)\end{array}$ & 159.1 & 167.1 & 168.2 & 173.8 & 0.0319 & 0.2646 & 0.1021 & 0.7985 \\
\hline OGHLW-W & $\begin{array}{c}\alpha \\
0.0062 \\
(0.0198)\end{array}$ & $\begin{array}{c}\beta \\
0.1375 \\
(0.2013)\end{array}$ & $\begin{array}{c}\lambda \\
9.8384 \\
(0.0027)\end{array}$ & $\begin{array}{c}\gamma \\
0.7102 \\
(0.4516)\end{array}$ & 160.6 & 168.6 & 169.8 & 175.4 & 0.0353 & 0.2524 & 0.0856 & 0.9310 \\
\hline $\mathrm{KwW}$ & $\begin{array}{c}a \\
0.3778 \\
(0.2433)\end{array}$ & $\begin{array}{c}b \\
21.9782 \\
(0.0873)\end{array}$ & $\begin{array}{c}\alpha \\
0.0630 \\
(0.0067)\end{array}$ & $\begin{array}{c}\beta \\
9.9157 \\
(6.3940)\end{array}$ & 164.3 & 172.3 & 173.5 & 179.1 & 0.0727 & 0.5442 & 0.1091 & 0.7278 \\
\hline ELOLLOW & $\begin{array}{c}\beta \\
3.2768 \\
(1.7673)\end{array}$ & $\begin{array}{c}\lambda \\
0.1209 \\
(0.0285)\end{array}$ & $\begin{array}{c}\theta \\
2.9438 \\
(1.9669)\end{array}$ & $\begin{array}{c}\gamma \\
3.4997 \\
(0.6303)\end{array}$ & 163.8 & 171.8 & 172.9 & 178.5 & 0.0636 & 0.4815 & 0.1017 & 0.8027 \\
\hline KOL-LLoG & $\begin{array}{c}\mathrm{a} \\
0.6799 \\
(0.0896)\end{array}$ & $\begin{array}{c}\mathrm{b} \\
64.6120 \\
\left(1.1962 \times 10^{-5}\right)\end{array}$ & $\begin{array}{c}\lambda \\
0.0002 \\
\left(9.0536 \times 10^{-5}\right)\end{array}$ & $\begin{array}{c}c \\
2.8882 \\
(0.0019)\end{array}$ & 165.1 & 173.1 & 174.2 & 179.8 & 0.0770 & 0.5729 & 0.1038 & 0.7821 \\
\hline BOL-E & $\begin{array}{c}a \\
0.2105 \\
(0.0371)\end{array}$ & $\begin{array}{c}\mathrm{b} \\
0.3251 \\
(0.2604)\end{array}$ & $\begin{array}{c}\lambda \\
0.0003 \\
(0.0002)\end{array}$ & $\begin{array}{c}\theta \\
1.1911 \\
(0.0088)\end{array}$ & 159.0 & 167.0 & 168.1 & 173.7 & 0.0343 & 0.2175 & 0.0832 & 0.9449 \\
\hline
\end{tabular}

\section{Concluding remarks}

We developed a new generalized distribution called the Marshall-Olkin-Gompertz-G (MO-Gom-G) family of distributions. We derived the statistical properties of the MO-Gom-G family of distributions. Maximum likelihood estimates of the model parameters were also derived. A simulation study was conducted to evaluate the consistency of the maximum likelihood estimates. A special case (MO-Gom-W) was applied to two real data examples to demonstrate the flexibility of the MO-Gom-G family of distributions.

\section{Acknowledgment}

We are grateful to the editor and referees for the improved version of this paper.

\section{References}

[1] M. Alizadeh, L. Benkhelifa, M. Rasekhi, B. Hosseini, The odd log-logistic generalized Gompertz distribution: Properties, applications and different methods of estimation, Commun. Math. Stat., 8 (2020), 295-317. 1

[2] M. Alizadeh, G. M. Cordeiro, L. G. Bastos Pinho, I. Ghosh, The Gompertz-G family of distributions, J. Stat. Theory Pract., 11 (2017), 179-207. 1, 2

[3] M. Alizadeh, S. Tahmasebi, M. R. Kazemi, H. S. A. Nejad, G. H. G. Hamedani, The odd log-logistic Gompertz lifetime distribution: Properties and applications, Studia Sci. Math. Hungar., 56 (2019), 55-80. 1

[4] W. Barreto-Souza, H. S. Bakouch, A New Lifetime Model with Decreasing Failure Rate, Statistics, 47 (2013), 465-476. 6

[5] W. Barreto-Souza, A. J. Lemonte, G. M. Cordeiro, General results for the Marshall and Olkin's family of distributions, An. Acad. Brasil. Ciênc., 85 (2013), 3-21. 2.3

[6] M. Bourguignon, R. B. Silva, G. M. Cordeiro, The Weibull-G family of probability distributions, J. Data Sci., 12 (2014), 53-68. 1, 6.1

[7] J. M. Chambers, W. S. Cleveland, B. Kleiner, P. A. Tukey, Graphical Methods of Data Analysis, Inter. Bio. Soc., 40 (1984), 567-568. 6

[8] F. Chipepa, B. Oluyede, B. Makubate, The Odd Generalized Half-Logistic Weibull-G Family of Distributions: Properties and Applications, Journal of Statistical Modelling: Theory and Applications, (2020), 1-25. 6 
[9] F. Chipepa, B. Oluyede, B. Makubate, A. Fagbamigbe, The Beta Odd Lindley-G Family of Distributions with Applications, J. Probab. Stat. Sci., 17 (2019), 51-83. 6

[10] F. Chipepa, B. Oluyede, B. Makubate, A New Generalized Family of Odd Lindley-G Distribution with application, Int. J. Stat. Probab., 8 (2019), 23 pages. 6

[11] F. Chipepa, B. Oluyede, D. Wanduku, The Exponentiated Half Logistic Odd Weibull-Topp-Leone-G Family of Distributions: Model, Properties and Applications, Journal of Statistical Modelling: Theory and Applications, (2020), (In Press). 6

[12] G. M. Cordeiro, M. Alizadeh, P. R. D. Marinho, The type I half-logistic family of distributions, J. Stat. Comput. Simul., 86 (2016), 707-728. 1

[13] G. M. Cordeiro, M. Alizadeh, A. D. C. Nascimento, M. Rasekhi, The Exponentiated Gompertz Generated Family of Distributions: Properties and Applications, Chil. J. Stat., 7 (2016), 29-50. 1

[14] G. M. Cordeiro, M. de Castro, A New Family of Generalized Distributions, J. Stat. Comput. Simul., 81 (2011), $883-898$. 1

[15] G. M. Cordeiro, E. M. M. Ortega, S. Nadarajaah, The Kumaraswamy Weibull Distribution with Application to Failure Data, J. Franklin Inst., 347 (2010), 1399-1429. 6

[16] A. El-Gohary, A. Alshamrani, A. N. Al-Otaibi, The Generalized Gompertz Distribution, Appl. Math. Model., 37 (2013), 13-24. 6

[17] N. Eugene, C. Lee, F. Famoye, Beta-Normal Distribution and Its Applications, Comm. Statist. Theory Methods, 31 (2002), 497-512. 1

[18] J. U. Gleaton, J. D. Lynch, Properties of Generalized Log-Logistic Families of Lifetime Distributions, JPSS J. Probab. Stat. Sci., 4 (2006), 51-64. 1

[19] B. Gompertz, On the Nature of the Function Expressive of the Law of Human Mortality and on the New Mode of Determining the Value of Life Contingencies, Philos. Trans. Roy. Stat. Soc., 115 (1825), 513-580. 6

[20] W. Gui, Marshall-Olkin Extended Log-logistic Distribution and Its Application in Minification Processes, Appl. Math. Sci., 7 (2013), 3947-3961. 6

[21] R. D. Gupta, D. Kundu, Generalized Exponential Distributions, Aust. N. Z. J. Stat., 41 (1999), 173-188. 1

[22] M. R. Gurvich, A. T. DiBenedetto, S. V. Ranade, A New Statistical Distribution for Characterizing the Random Strength of Brittle Materials, J. Mater. Sci., 32 (1997), 2559-2564. 1

[23] A. A. Jafari, S. Tahmasebi, M. Alizadeh, The beta-Gompertz distribution, Rev. Colombiana Estadíst., 37 (2014), 141158. 1

[24] H. Karamikabir, M. Afshari, M. Alizadeh, G. G. Hamedani, A new extended generalized Gompertz distribution with statistical properties and simulations, Comm. Statist. Theory Methods, (2019), 1-29. 1

[25] M. Ç. Korkmaz, H. M. Yousof, G. G. Hamedani, The Exponential Lindley Odd Log-Logistic-G family: Properties, Characterizations and Applications, J. Stat. Theory Appl., 17 (2018), 554-571. 6

[26] A. W. Marshall, I. Olkin, A New Method for Adding a Parameter to a Family of Distributions with Applications to the Exponential and Weibull Families, Biometrika, 84 (1997), 641-652. 1, 1, 2

[27] R. M. Pakungwati, Y. Widyaningsih, D. Lestari, Marshall-Olkin Extended Inverse Weibull Distribution and Its Application, J. Phys.: Conf. Ser., 1108 (2018), 7 pages. 6

[28] R. C. Team, $R$ a Language and Environment for Statistical Computing, (2013). 6

[29] A. Rényi, On Measures of Entropy and Information, Univ. California Press, Berkeley, Calif., (1960) 547-561. 2.5

[30] M. M. Ristić, N. Balakrishnan, The Gamma Exponentiated Exponential Distribution, J. Stat. Comput. Simul., 82 (2012), 1191-1206. 1

[31] M. Shaked, J. G. Shanthikumar, Stochastic Orders, Springer, New York, (2007). 2.2

[32] C. E. Shannon, Prediction and Entropy of Printed English, Bell Syst. Tech. J., 30 (1951), 50-64. 2.5

[33] R. L. Smith, J. C. Naylor, A Comparison of Maximum Likelihood and Bayesian Estimators for the Three-Parameter Weibull Distribution, Appl. Statist., 36 (1987), 358-369. 6.1

[34] H. Torabi, N. M. Hedesh, The Gamma-Uniform Distribution and Its Applications, Kybernetika, 48 (2012), 16-30. 1

[35] K. Xu, M. Xie, L. C. Tang, S. L. Ho, Application of Neural Networks in Forecasting Engine Systems reliability, Appl. Soft. Comput, 2 (2003), 255-268. 6.2

[36] K. Zografos, N. Balakrishnan, On Families of Beta- and Generalized Gamma-Generated Distributions and Associated Inference, Stat. Methodol., 6 (2009), 344-362. 1

\section{Appendix}

The following url contains the simulation algorithm for the MO-Gom-W distribution https://drive.google. com/file/d/1e3yWmgmoMHzYLrMOanFVJ7BjNjIubnLg/view?usp=sharing. 